\title{
An ICON Overview on Physical Modalities for Neck Pain and Associated Disorders
}

\author{
Nadine Graham ${ }^{*}, 1$, Anita R Gross ${ }^{1}$, Lisa C. Carlesso ${ }^{2}$, P. Lina Santaguida ${ }^{3}$, Joy C MacDermid ${ }^{4}$, \\ Dave Walton ${ }^{5}$, Enoch $\mathrm{Ho}^{1}$ and $\mathrm{ICON}^{\S}$ \\ ${ }^{1}$ School of Rehabilitation Science, McMaster University, Hamilton, Ontario, Canada \\ ${ }^{2}$ Department of Clinical Epidemiology and Biostatistics, McMaster University, Hamilton, Ontario, Canada \\ ${ }^{3}$ University Evidence-based Practice Centre, Department of Clinical Epidemiology and Biostatistics, Hamilton, Ontario, \\ Canada \\ ${ }^{4}$ School of Rehabilitation Sciences, McMaster University, Hamilton, Ontario and Hand and Upper Limb Centre, St. Joseph's \\ Health Centre, London, Ontario, Canada \\ ${ }^{5}$ School of Physical Therapy, University of Western Ontario, London, Ontario, Canada
}

\begin{abstract}
Introduction: Neck pain is common, can be disabling and is costly to society. Physical modalities are often included in neck rehabilitation programs. Interventions may include thermal, electrotherapy, ultrasound, mechanical traction, laser and acupuncture. Definitive knowledge regarding optimal modalities and dosage for neck pain management is limited.

Purpose: To systematically review existing literature to establish the evidence-base for recommendations on physical modalities for acute to chronic neck pain.

Methods: A comprehensive computerized and manual search strategy from January 2000 to July 2012, systematic review methodological quality assessment using AMSTAR, qualitative assessment using a GRADE approach and recommendation presentation was included. Systematic or meta-analyses of studies evaluating physical modalities were eligible. Independent assessment by at least two review team members was conducted. Data extraction was performed by one reviewer and checked by a second. Disagreements were resolved by consensus.

Results: Of 103 reviews eligible, 20 were included and 83 were excluded. Short term pain relief - Moderate evidence of benefit: acupuncture, intermittent traction and laser were shown to be better than placebo for chronic neck pain. Moderate evidence of no benefit: pulsed ultrasound, infrared light or continuous traction was no better than placebo for acute whiplash associated disorder, chronic myofascial neck pain or subacute to chronic neck pain. There was no added benefit when hot packs were combined with mobilization, manipulation or electrical muscle stimulation for chronic neck pain, function or patient satisfaction at six month follow-up.

Conclusions: The current state of the evidence favours acupuncture, laser and intermittent traction for chronic neck pain. Some electrotherapies show little benefit for chronic neck pain. Consistent dosage, improved design and long term follow-up continue to be the recommendations for future research.
\end{abstract}

Keywords: Neck pain, review of reviews, modalities, knowledge synthesis.

\begin{abstract}
*Address correspondence to this author at the School of Rehabilitation Science, IAHS, 1400 Main Street West, 4th Floor, Hamilton, ON, L8S 1C7, Canada; Tel: 905-525-9140, Ext. 22524; Fax: 905-304-4431;

E-mail: ngraham@mcmaster.ca
\end{abstract}

${ }^{\S}$ ICON is a multi-disciplinary collaborative group that includes scientist-authors (listed below) and support staff (Margaret Lomotan) that conduct knowledge synthesis and translation aimed at reducing the burden of neck pain.

The ICON authors that provided direction of the project and reviewed the findings/manuscript include (in alphabetical order): Gert Bronfort, Norm Buckley, Lisa Carlesso, Linda Carroll, Pierre Côté, Jeanette Ezzo, Paulo Ferreira, Tim Flynn, Charlie Goldsmith, Anita Gross, Ted Haines, Jan Hartvigsen, Wayne Hing, Gwendolen Jull, Faith Kaplan, Ron Kaplan, Helge Kasch, Justin Kenardy, Per Kjær, Janet Lowcock, Joy MacDermid, Jordan Miller, Margareta Nordin, Paul Peloso, Jan Pool, Duncan Reid, Sidney Rubinstein, P. Lina Santaguida, Anne Söderlund, Natalie Spearing, Michele Sterling, Grace Szeto, Robert Teasell, Arianne Verhagen, David M. Walton, Marc White.

Expert Panel: Gert Bronfort DC, PhD, Northwestern Health Sciences University, USA; Duncan Reid PT, PhD, School Rehabilitation and Occupation Studies AUT University, NZ; Ron Kaplan Psychologist, PhD, Canada; Anne Söderlund PT, PhD, School of Health, Care and Social Welfare Malardalens University, Sweden; Grace P.Y. Szeto PT, PhD, Department of Rehabilitation Sciences Hong Kong Polytechnic University, Hong Kong; Marc White MD, Canadian Institute for the Relief of Pain and Disability, Canada.

\section{INTRODUCTION}

\section{Description of the Condition}

Neck pain is common, can be disabling and is costly to society. Twenty-six to $71 \%$ of the adult population can recall experiencing an episode of neck pain or stiffness in their lifetime [1-3]. Although most people with neck disorders experience a low level of disability, Cote 1998 found that $5 \%$ were significantly disabled. The prevalence of neck pain is higher in females [4-7]. The results of The Bone and Joint Decade 2000-2010 Task Force on Neck Pain reveal 12month neck pain prevalence estimates ranging from $30 \%$ to $50 \%$ in the adult general population generally rising to middle age and then declining in later life [7]. In a U.S. study from the National Ambulatory Medical Care Survey, an average of 10.2 million visits to health care facilities for neck pain was reported [8]. Neck pain has a large impact on health care expenditure, attributed to visits to healthcare 
providers, sick leave and is responsible for significant disability and loss of productivity [9-11].

\section{Description of the Intervention}

The primary approach to neck pain is conservative management. Physical modalities are often a component of these management programs. Interventions may include various forms of heat and cold application, electrotherapy, ultrasound, mechanical traction, laser and acupuncture. Ultrasound is one of the most widely used modalities yet conflicting or limited evidence exists regarding its effectiveness [12, 13]. Previous to this current overview, Gross 2002 [14] and Spitzer 1995 [15] found thermal agents to be commonly used in addition to manual therapy, exercise, education and drug therapies for acute and chronic neck pain. Since physical agents are used as adjunctive interventions, it can be difficult to determine what contribution they make to augmenting treatment effects. Previous reviews have questioned the benefit of physical agents. Gross 2007 [16] found evidence of no benefit for the use of hot packs for both intermediate and long-term relief of chronic pain or improved function. In addition, infrared light and spray and stretch did not aid in short-term pain reduction. Two systematic reviews, one examining noninvasive treatment for trigger point pain [17] and the other on conservative treatment for acute neck pain not due to whiplash [18] did not report on any studies that included heat or cold therapy. Electrotherapy [Electrical Nerve Stimulation, Electrical Muscle Stimulation, Pulsed Electrical Magnetic Field] has been commonly used as one of the physiotherapeutic options to treat neck pain for many years [19]. Little is known about the efficacy of most of these subtypes as sound empirical evidence is lacking. An updated Cochrane review [19] still could not evaluate the unique contribution of electrotherapy since studies had not examined their effects in isolation. Mechanical traction is another treatment with limited evidence of effectiveness [2024]. According to a number of existing reviews, moderate evidence suggests that acupuncture is effective in the short term for relieving neck pain [16, 25-28]. LASER is a conservative method of treating neck pain that has received relatively limited attention in the scientific literature to date.

\section{How the Interventions Might Work}

We consider the different physiological and clinical rationale for the use of various physical modalities used by clinicians.

\section{HEAT AND COLD}

Heat and cold can be applied in multiple ways ranging from in-clinic devices to home applications, with varying thermal properties that can influence physiological effects. Therapeutic applications may include cold packs, evaporative cooling spray, superficial moist heat, shortwave diathermy, infrared heat and hydrotherapy. These therapies can assist in the healing process by providing physiological changes to a range of tissues [29] including changes in blood flow, nerve conduction, and metabolic function. Since the biophysical properties differ across cold and heat agents, the capability of reaching particular target tissues varies across modalities [29-32].

\section{ULTRASOUND}

Therapeutic ultrasound is a form of acoustic energy (sound) that has been used in rehabilitative medicine for over fifty years [33]. It is used for the purpose of stimulating soft tissue repair and inflammation management thereby resulting in the relief of pain [34] and also for bone healing [35]. Ultrasound is considered a 'deep heating modality' as it is able to increase the temperature of tissues at a much greater depth than superficial heat through the mechanical effects of sound vibration. It should be noted however, that ultrasound can be used without producing a significant rise in tissue temperature [34]. It is believed that ultrasound application increases blood flow and metabolism at the site of injury, and can thereby decrease pain and increase the rate of healing [33, 34].

\section{MECHANICAL TRACTION}

Mechanical traction for the cervical spine involves a longitudinal force applied to the neck via a mechanical system that is delivered intermittently or continuously [36]. It is often used as an adjunct therapy in outpatient rehabilitation [29]. The physiological effects of mechanical traction for the cervical spine may include separation of vertebral bodies, movement of facet joints, expansion of intervertebral foramen and stretching of soft tissue [29, 36].

\section{ELECTROTHERAPY}

Electrotherapy treatment may include: Direct current (DC), iontophoresis, electrical nerve stimulation; electrical muscle stimulation; transcutaneous electrical nerve stimulation (TENS); pulsed electromagnetic fields, repetitive magnetic stimulation and permanent magnets (albeit extremely small current). Treatment by DC or Galvanic current, reduces pain by inhibiting nociceptor activity [37]. The main indication for Galvanic current is the treatment of acute radicular pain and inflammation of periarticular structures such as tendons and ligaments. Alternating electrical current (AC) or modulated DC (Galvanic stimulation) may be effective by inhibiting pain-related potentials on the spinal and supraspinal level. Pain relief can be obtained through possible endorphinergic mechanisms of analgesia with the use of electrical muscle stimulation, TENS, or other forms of electrical nerve stimulation [38].

\section{ACUPUNCTURE}

Acupuncture has been increasingly used as an alternative to more traditional treatments for musculoskeletal pain. It is defined as the stimulation of a certain point(s) on the body, by the insertion of needles, to achieve a desirable effect. It is believed to prevent or modify the perception of pain or to alter physiological functions, including pain control for the treatment of certain diseases or dysfunction of the body [39]. One theory from western scientific research suggests that acupuncture promotes the release of endorphins from the brain through the stimulation of peripheral nerves. These endorphins then block pain pathways in the brain [40]. Traditional Chinese Medicine tracing back thousands of years, encompasses the ancient philosophy of Taoism and the concept of universal balance between Yin and Yang. To balance one's energy by either sedating or stimulating acupuncture points, promoting the flow of Qi (life's energy) and thereby restoring health $[41,42]$. 
We acknowledge and respect the variations in the underpinning theory and practical application of acupuncture and related challenges it poses to our evaluation of this intervention and potential subtypes.

\section{LASER}

The term LASER is an acronym for light amplification by stimulated emission of radiation-a form of photonic therapy that uses monochromatic light with either high or low power [34]. Low power LASER devices have little to no thermal effects and are used to treat an array of musculoskeletal conditions to decrease pain, inflammation and soft tissue scars, and to promote fracture healing [34, 4345]. Three main types of LASER are used clinically: heliumneon (HeNe wavelength $632.8 \mathrm{~nm}$, tissue penetration $0.8 \mathrm{~mm}$ ), the gallium-arsenide (GaAs $904 \mathrm{~nm}$, penetrating $5 \mathrm{~cm}$ ) and the gallium-aluminum-arsenide (GaAlAs 830nm, penetrating 2-3cm) [34, 43]. Many mechanisms of action have been proposed for LASER including the slowing of transmission of nociceptive signals, regulation of serotonin and norepinephrine and may limit the release of proinflammatory mediators [34, 46-48].

\section{MULTIMODAL TREATMENTS}

Multimodal treatment plans are common in outpatient rehabilitation with physical modalities often used as a component of the total intervention. Modalities are typically not used exclusively. For that reason, many randomized trials do not examine the use of modalities in isolation but rather in combination with other treatments such as exercise or manual therapy. It is common to see one group of interventions compared to a completely different set of combined interventions. These types of studies may determine a treatment grouping that is more effective than another. Although this makes clinical sense, the independent contribution of a particular modality alone cannot be evaluated and designs that might disaggregate the separate treatment effects are rarely used [49]. Clinically, these various modalities are used for improving physiologic functions that promote healing; or, short-term pain reduction, improved joint and muscle motion. Also, modalities are sometimes used prior to exercise to improve exercise tolerance.

\section{Why it is Important to do this Overview}

Conflicting or unclear evidence regarding the effectiveness of physical modalities for whiplash-associated disorders (WAD) remains prominent in the literature due in part to poor methodological quality of efficacy trials [23, 50]. Despite more recent studies over the last decade with sound methodology, the best evidence for treatment of WAD patients remains relatively unchanged [51]. The evidence for treatment of cervicogenic headache and radiculopathy are underrepresented in the current literature and there is no evidence of the optimal dosage of non-invasive treatments [51]. Chronic neck pain accounts for \$150-\$200 billion US each year in economic loss due to lost work days and rehabilitation, yet relatively little is known about how best to manage this condition $[16,52,53]$. Overall, limited definitive knowledge as to what modalities including dosage is most effective to address the management of neck pain.

The purpose of this overview was to systematically review existing reviews and to establish the evidence-base recommendations on the treatment of acute to chronic neck pain (specific and non-specific) with physical modalities. These included heat and cold application, electrotherapy, ultrasound, mechanical traction, laser and acupuncture. We specifically focused on evidence that evaluated use of physical modalities for reducing pain and improving function, quality of life, patient satisfaction or global perceived effect compared to a control with immediately post treatment to long term follow up.

\section{METHODS}

Our systematic overview process included comprehensive computerized search strategies including MEDLINE, EMBASE, CINAHL, ILC, CENTRAL and LILACS from January 2000 to August 2010, selection criteria (Table 1). Independent assessments by at least two members of our review team were performed for a systematic review of methodological quality using the AMSTAR tool [54], qualitative assessment of the strength of evidence using the Grading of Recommendations, Assessment, Development and Evaluation (GRADE) approach [55, 56] and the recommendation presentation. Two separate searches were performed, one for treatment and one for harms. This methodology is detailed in our International Collaboration on Neck (ICON) methods report

Table 1. Inclusion and Exclusion Criteria Set a Priori

\begin{tabular}{|c|c|}
\hline PICOSS & Criteria \\
\hline Participant & Adult ( $\geq 18$ year), acute to chronic neck pain with or without cervicogenic headache or radiculopathy or whiplash \\
\hline Intervention & Acupuncture, electrotherapy, laser, cold or heat, mechanical traction, ultrasound as single treatment \\
\hline Comparison & Control or comparison (i.e. standard care, another treatment) \\
\hline Outcomes & $\begin{array}{l}\text { Primary: Pain, function, disability, work related, quality of life } \\
\text { Secondary: global perceived effect and patient satisfaction }\end{array}$ \\
\hline Study Design & Systematic reviews of randomized trials; narrative reviews were excluded \\
\hline $\begin{array}{l}\text { Study } \\
\text { Timeframe }\end{array}$ & $\begin{array}{l}\text { Immediate post-treatment (IP), short-term (ST: closest to } 3 \text { months); intermediate term (IT: closest to } 6 \text { months; long term (LT: closest } \\
\text { to } 1 \text { year) }\end{array}$ \\
\hline
\end{tabular}


[57] including full search terms; this protocol was not registered. Further, we complemented this search by identifying on-going systematic reviews near completion such as Cochrane Reviews up to July 2012, by contacting our expert panel and by systematically checking reference lists of primary studies to minimize the risk of missing relevant reviews and trials.

Data extraction was performed using pre-piloted forms by one reviewer and checked by a second with disagreements resolved by consensus. We systematically extracted data from selected reviews and developed evidence tables. Extractable data from the reviews included the following: author, year of publication, disorder type with duration of symptoms, the intervention, the type of comparator (placebo, no care, usual care, other treatment), the search period, the original authors of primary studies, the AMSTAR score, the effect direction, effect size for reported primary outcomes with duration follow-up period, reported harms, quality ranking system, evidence statement and final GRADE. Harm information was summarized qualitatively from both the treatment reviews that reported any adverse events and directly from the harms reviews.

We utilized the following triage rules (set a priori) to guide decisions and to group treatment reviewed:

1) Type of treatment was used to group reviews by physical modality (heat and cold application, electrotherapy, ultrasound, mechanical traction, laser and acupuncture).

2) Within a treatment modality we grouped review data with respect to comparator treatments.

3) Once the studies had been grouped by these two categories, a decision was made PER grouping; if there were few reviews within a treatment category and with unique comparators, we did not eliminate any further reviews.

4) If there were several reviews on the same treatment and comparator, we prioritized to obtain the best quality reviews by considering the approach recommended by Whitlock et al. [58] as follows:

A. Year of publication. Within the group of systematic reviews

i. If there were reviews that were very similar across multiple years, we focused on reviews that were the most up-to-date AND if the studies included in the older review were also INCLUDED in the more recent review.

ii. We cross-checked to ascertain that the conclusions were similar to the more current systematic review.

B. AMSTAR- Risk of Bias. Reviews were considered low risk of bias if they scored 8 or higher on the 11-point AMSTAR scale, moderate risk of bias if scored between 5 and 7 , and high risk of bias if scored 4 or under. We used this process to focus on the best quality reviews. These were synthesized in a summary of findings table to help provide definitive summaries to inform clinical practice (Table 2). Inconsistency and discordance were highlighted and discussed in our methods paper [57].

5) Effect Size estimates: We selected the effect size as the primary summary measure for our overview. We determined that within a grouping for treatment and comparator, we selected a review to represent the BEST estimate of effect size or related meta-analysis and as needed reported the range of estimates for other included reviews. In cases where there was discordance between reviews, we reported the combined results of the individual included studies. Additional data on magnitude of effect such as number-needed-to-treat (NNT) and weighted mean difference (WMD) were extracted when available.

6) Strength of Evidence using GRADE approach: We used this same representative systematic review or meta-analysis from which to judge an overall GRADE for the strength of the body of evidence for treatment. This did not include the harms evaluation that was observational. The selected reviews may have already had a GRADE table. We estimated the quality of the evidence using the GRADE approach for primary trials within reviews using reported information on: design [randomized controlled trials (RCT), immediately post treatment to long term follow-up]; risk of bias (equivalent methodological criteria for risk of bias reported in the review i.e. JADAD [59] or PEDro [60]); imprecision (sample size); inconsistency; indirectness; and reporting bias.

We excluded reviews that did not meet our inclusion criteria with rationale for exclusion. Multimodal treatments were not included if contributions of individual interventions could not be determined. Once reviews were deemed relevant and of at lower risk of bias, we extracted and reported individual trial findings by "overall strength of evidence" using GRADE approach and stratified by "treatment category" for the Summary of Findings Table (Table 3). Conflicting evidence was recorded (Table 4). The final core recommendations are reported in Evidence-Based Recommendations (Table 5).

\section{RESULTS}

From 117 reviews that were identified for the entire ICON treatment category, 103 were screened for eligibility. From these, a total of 48 reviews evaluated the physical modalities of interest, with 20 reviews included (Fig. 1 PRISMA diagram). Eighty-three reviews were excluded, detailed reasons can be found in APPENDIX 1.

Those systematic reviews that scored less than six on the AMSTAR assessment were not included. The AMSTAR assessment disclosed that the most common methodological limitations included incomplete reporting on: publication bias; conflict of interest; and complete reporting of excluded studies [57]. The primary reviews included in our analyses evaluated the evidence on the following physical modalities: thermal, electrotherapy, ultrasound, mechanical traction, laser and acupuncture. Treatment parameters varied across interventions and some were not reported at all. Treatment dosages have been reported when available. 
Table 2. AMSTAR Rating

\begin{tabular}{|c|c|c|c|c|c|c|c|c|c|c|c|c|}
\hline Ref\# & Author & 1 & 2 & 3 & 4 & 5 & 6 & 7 & 8 & 9 & 10 & 11 \\
\hline 107 & Baxter et al. 2008 & $\mathrm{Y}$ & $\mathrm{N}$ & $\mathrm{Y}$ & $\mathrm{N}$ & $\mathrm{N}$ & $\mathrm{Y}$ & $\mathrm{Y}$ & $\mathrm{Y}$ & NA & $\mathrm{N}$ & $\mathrm{N}$ \\
\hline 157 & Bronfort et al. 2010 & $\mathrm{Y}$ & $\mathrm{N}$ & $\mathrm{Y}$ & $\mathrm{N}$ & $\mathrm{N}$ & $\mathrm{N}$ & $\mathrm{Y}$ & $\mathrm{Y}$ & NA & $\mathrm{N}$ & $\mathrm{N}$ \\
\hline 1737 & Chow \& Barnsley 2005 & $\mathrm{Y}$ & $\mathrm{N}$ & $\mathrm{Y}$ & $\mathrm{Y}$ & $\mathrm{Y}$ & $\mathrm{Y}$ & $\mathrm{Y}$ & $\mathrm{N}$ & NA & $\mathrm{N}$ & $\mathrm{N}$ \\
\hline 1747 & Conlin et al. 2005 & $\mathrm{Y}$ & $\mathrm{N}$ & $\mathrm{Y}$ & $\mathrm{N}$ & $\mathrm{N}$ & $\mathrm{Y}$ & $\mathrm{Y}$ & $\mathrm{Y}$ & $\mathrm{Y}$ & $\mathrm{N}$ & $\mathrm{N}$ \\
\hline 25234 & Ernst et al. 2011 & $\mathrm{Y}$ & $\mathrm{CA}$ & $\mathrm{Y}$ & $\mathrm{N}$ & $\mathrm{N}$ & $\mathrm{Y}$ & $\mathrm{N}$ & NA & NA & $\mathrm{N}$ & $\mathrm{N}$ \\
\hline 106 & Fu et al. 2009 & $\mathrm{Y}$ & $\mathrm{Y}$ & $\mathrm{Y}$ & $\mathrm{N}$ & $\mathrm{Y}$ & $\mathrm{Y}$ & $\mathrm{Y}$ & $\mathrm{N}$ & $\mathrm{Y}$ & $\mathrm{N}$ & $\mathrm{N}$ \\
\hline 20018 & Furlan et al. 2010 & $\mathrm{Y}$ & $\mathrm{Y}$ & $\mathrm{Y}$ & $\mathrm{Y}$ & $\mathrm{Y}$ & $\mathrm{Y}$ & $\mathrm{Y}$ & $\mathrm{Y}$ & $\mathrm{Y}$ & $\mathrm{N}$ & $\mathrm{N}$ \\
\hline 69 & Gross et al. 2007 & $\mathrm{Y}$ & Y & $\mathrm{Y}$ & $\mathrm{Y}$ & $\mathrm{N}$ & $\mathrm{Y}$ & $\mathrm{Y}$ & $\mathrm{Y}$ & $\mathrm{Y}$ & $\mathrm{N}$ & $\mathrm{N}$ \\
\hline 5 & Gross et al. 2010 & $\mathrm{Y}$ & $\mathrm{Y}$ & $\mathrm{Y}$ & $\mathrm{Y}$ & $\mathrm{Y}$ & $\mathrm{Y}$ & $\mathrm{Y}$ & $\mathrm{Y}$ & $\mathrm{Y}$ & $\mathrm{N}$ & $\mathrm{N}$ \\
\hline 20041 & Gross et al. 2012 & $\mathrm{Y}$ & $\mathrm{Y}$ & $\mathrm{Y}$ & $\mathrm{Y}$ & $\mathrm{N}$ & $\mathrm{Y}$ & $\mathrm{Y}$ & Y & Y & $\mathrm{Y}$ & $\mathrm{N}$ \\
\hline 36 & Haines et al. 2009 & $\mathrm{Y}$ & $\mathrm{Y}$ & $\mathrm{Y}$ & $\mathrm{Y}$ & $\mathrm{Y}$ & $\mathrm{Y}$ & $\mathrm{Y}$ & $\mathrm{Y}$ & $\mathrm{Y}$ & $\mathrm{N}$ & $\mathrm{N}$ \\
\hline 83 & Haraldsson et al. 2006 & $\mathrm{Y}$ & $\mathrm{Y}$ & $\mathrm{Y}$ & $\mathrm{Y}$ & $\mathrm{Y}$ & $\mathrm{Y}$ & $\mathrm{Y}$ & $\mathrm{Y}$ & $\mathrm{Y}$ & $\mathrm{N}$ & $\mathrm{N}$ \\
\hline 53 & Hurwitz et al. 2008 & $\mathrm{Y}$ & $\mathrm{N}$ & $\mathrm{N}$ & $\mathrm{Y}$ & $\mathrm{N}$ & $\mathrm{Y}$ & $\mathrm{Y}$ & $\mathrm{Y}$ & NA & $\mathrm{N}$ & $\mathrm{N}$ \\
\hline 193 & Itoh \& Kitakoji 2007 & $\mathrm{Y}$ & $\mathrm{Y}$ & $\mathrm{Y}$ & $\mathrm{N}$ & $\mathrm{N}$ & $\mathrm{Y}$ & $\mathrm{Y}$ & $\mathrm{Y}$ & NA & $\mathrm{N}$ & $\mathrm{N}$ \\
\hline 166 & Kay et al. 2009 & $\mathrm{Y}$ & $\mathrm{Y}$ & $\mathrm{Y}$ & $\mathrm{Y}$ & $\mathrm{Y}$ & $\mathrm{Y}$ & $\mathrm{Y}$ & $\mathrm{Y}$ & $\mathrm{Y}$ & $\mathrm{N}$ & $\mathrm{N}$ \\
\hline 145 & Lin et al. 2009 & $\mathrm{Y}$ & $\mathrm{Y}$ & $\mathrm{Y}$ & $\mathrm{N}$ & $\mathrm{Y}$ & $\mathrm{CA}$ & $\mathrm{N}$ & NA & $\mathrm{N}$ & $\mathrm{N}$ & $\mathrm{N}$ \\
\hline 303278 & Lin et al. 2012 & $\mathrm{Y}$ & $\mathrm{Y}$ & $\mathrm{Y}$ & $\mathrm{Y}$ & $\mathrm{Y}$ & $\mathrm{Y}$ & $\mathrm{Y}$ & $\mathrm{Y}$ & NA & $\mathrm{N}$ & $\mathrm{N}$ \\
\hline 6020 & Miller et al. 2010 & $\mathrm{Y}$ & $\mathrm{Y}$ & $\mathrm{Y}$ & $\mathrm{Y}$ & $\mathrm{N}$ & $\mathrm{Y}$ & $\mathrm{Y}$ & $\mathrm{Y}$ & $\mathrm{Y}$ & $\mathrm{N}$ & $\mathrm{N}$ \\
\hline 3333 & Peake \& Harte, 2005 & $\mathrm{Y}$ & $\mathrm{N}$ & $\mathrm{Y}$ & $\mathrm{N}$ & $\mathrm{N}$ & $\mathrm{Y}$ & $\mathrm{Y}$ & $\mathrm{Y}$ & NA & $\mathrm{N}$ & $\mathrm{N}$ \\
\hline 495 & Peloso et al. 2007 & $\mathrm{Y}$ & $\mathrm{Y}$ & $\mathrm{Y}$ & $\mathrm{Y}$ & $\mathrm{Y}$ & $\mathrm{Y}$ & $\mathrm{Y}$ & $\mathrm{Y}$ & $\mathrm{Y}$ & $\mathrm{Y}$ & $\mathrm{N}$ \\
\hline 1432 & Reid \& Rivett 2005 & $\mathrm{Y}$ & $\mathrm{CA}$ & $\mathrm{Y}$ & $\mathrm{N}$ & $\mathrm{N}$ & $\mathrm{Y}$ & $\mathrm{Y}$ & $\mathrm{Y}$ & NA & $\mathrm{N}$ & $\mathrm{N}$ \\
\hline 226 & Rickards 2006 & $\mathrm{Y}$ & $\mathrm{N}$ & $\mathrm{Y}$ & $\mathrm{N}$ & $\mathrm{Y}$ & $\mathrm{Y}$ & $\mathrm{Y}$ & $\mathrm{Y}$ & NA & $\mathrm{N}$ & $\mathrm{N}$ \\
\hline 241 & Teasell et al. 2010 & $\mathrm{Y}$ & $\mathrm{N}$ & $\mathrm{Y}$ & $\mathrm{N}$ & $\mathrm{N}$ & $\mathrm{Y}$ & $\mathrm{Y}$ & $\mathrm{Y}$ & NA & $\mathrm{N}$ & $\mathrm{N}$ \\
\hline 11690 & Teasell et al. 2010 & $\mathrm{Y}$ & $\mathrm{N}$ & $\mathrm{Y}$ & $\mathrm{N}$ & $\mathrm{N}$ & $\mathrm{Y}$ & $\mathrm{Y}$ & $\mathrm{Y}$ & NA & $\mathrm{N}$ & $\mathrm{N}$ \\
\hline 25360 & Trinh et al. 2006 & $\mathrm{Y}$ & $\mathrm{Y}$ & $\mathrm{Y}$ & $\mathrm{Y}$ & $\mathrm{Y}$ & $\mathrm{Y}$ & $\mathrm{Y}$ & $\mathrm{Y}$ & $\mathrm{Y}$ & $\mathrm{N}$ & $\mathrm{N}$ \\
\hline 213 & Trinh et al. 2007 & $\mathrm{Y}$ & $\mathrm{Y}$ & $\mathrm{Y}$ & $\mathrm{Y}$ & $\mathrm{Y}$ & $\mathrm{N}$ & $\mathrm{Y}$ & $\mathrm{Y}$ & $\mathrm{Y}$ & $\mathrm{N}$ & $\mathrm{N}$ \\
\hline 170 & Tsakitzidis et al. 2009 & $\mathrm{Y}$ & $\mathrm{Y}$ & $\mathrm{Y}$ & $\mathrm{Y}$ & $\mathrm{N}$ & $\mathrm{Y}$ & $\mathrm{Y}$ & $\mathrm{Y}$ & NA & $\mathrm{N}$ & $\mathrm{N}$ \\
\hline 185 & Verhagen et al. 2007 & $\mathrm{Y}$ & $\mathrm{Y}$ & $\mathrm{Y}$ & $\mathrm{N}$ & $\mathrm{Y}$ & $\mathrm{Y}$ & $\mathrm{Y}$ & $\mathrm{Y}$ & $\mathrm{Y}$ & $\mathrm{N}$ & $\mathrm{N}$ \\
\hline 75 & Vernon \& Humphreys 2007 & $\mathrm{Y}$ & $\mathrm{N}$ & $\mathrm{Y}$ & $\mathrm{N}$ & $\mathrm{N}$ & $\mathrm{Y}$ & $\mathrm{Y}$ & $\mathrm{Y}$ & NA & $\mathrm{N}$ & $\mathrm{N}$ \\
\hline 413 & Vernon \& Schneider 2009 & $\mathrm{Y}$ & $\mathrm{N}$ & $\mathrm{Y}$ & $\mathrm{N}$ & $\mathrm{N}$ & $\mathrm{N}$ & $\mathrm{Y}$ & $\mathrm{Y}$ & NA & $\mathrm{N}$ & $\mathrm{N}$ \\
\hline 1736 & Vernon et al. 2005 & $\mathrm{Y}$ & $\mathrm{CA}$ & $\mathrm{Y}$ & $\mathrm{N}$ & $\mathrm{N}$ & $\mathrm{Y}$ & $\mathrm{Y}$ & $\mathrm{Y}$ & NA & $\mathrm{N}$ & $\mathrm{N}$ \\
\hline
\end{tabular}




(Table 2) contd.....
\begin{tabular}{|c|c|c|c|c|c|c|c|c|c|c|c|c|}
\hline Ref\# & Author & $\mathbf{1}$ & $\mathbf{2}$ & $\mathbf{3}$ & $\mathbf{4}$ & $\mathbf{5}$ & $\mathbf{6}$ & $\mathbf{7}$ & $\mathbf{8}$ & $\mathbf{9}$ & $\mathbf{1 0}$ & $\mathbf{1 1}$ \\
\hline \hline 511 & Vernon et al. 2006 & $\mathrm{Y}$ & $\mathrm{N}$ & $\mathrm{Y}$ & $\mathrm{N}$ & $\mathrm{Y}$ & $\mathrm{Y}$ & $\mathrm{Y}$ & $\mathrm{N}$ & $\mathrm{NA}$ & $\mathrm{N}$ & $\mathrm{N}$ \\
\hline 71 & Vernon et al. 2007 & $\mathrm{Y}$ & $\mathrm{Y}$ & $\mathrm{Y}$ & $\mathrm{N}$ & $\mathrm{Y}$ & $\mathrm{Y}$ & $\mathrm{Y}$ & $\mathrm{Y}$ & $\mathrm{NA}$ & $\mathrm{N}$ & $\mathrm{N}$ \\
\hline 473 & Wang et al. 2008 & $\mathrm{Y}$ & $\mathrm{Y}$ & $\mathrm{Y}$ & $\mathrm{N}$ & $\mathrm{Y}$ & $\mathrm{Y}$ & $\mathrm{Y}$ & $\mathrm{Y}$ & $\mathrm{Y}$ & $\mathrm{N}$ & $\mathrm{N}$ \\
\hline 99 & Wang et al. 2009 & $\mathrm{Y}$ & $\mathrm{Y}$ & $\mathrm{N}$ & $\mathrm{N}$ & $\mathrm{N}$ & $\mathrm{Y}$ & $\mathrm{N}$ & $\mathrm{NA}$ & $\mathrm{NA}$ & $\mathrm{N}$ & $\mathrm{N}$ \\
\hline 57 & Williams et al. 2007 & $\mathrm{Y}$ & $\mathrm{Y}$ & $\mathrm{Y}$ & $\mathrm{N}$ & $\mathrm{N}$ & $\mathrm{Y}$ & $\mathrm{Y}$ & $\mathrm{Y}$ & $\mathrm{Y}$ & $\mathrm{N}$ & $\mathrm{N}$ \\
\hline
\end{tabular}

Key: Y-Yes; N -No; NA-not applicable; CA-can`t assess AMSTAR Questions:

1. Was an 'a priori' design provided? The research question and inclusion criteria should be established before the conduct of the review.

2. Was there duplicate study selection and data extraction? There should be at least two independent data extractors and a consensus procedure for disagreements should be in place.

3. Was a comprehensive literature search performed? At least two electronic sources should be searched. The report must include years and databases used (e.g. Central, EMBASE, and MEDLINE). Key words and/or MESH terms must be stated and where feasible.

4. Was the status of publication (i.e. grey literature) used as an inclusion criterion? The authors should state that they searched for reports regardless of their publication type. The authors should state whether or not they excluded any reports.

5. Was a list of studies (included and excluded) provided? A list of included and excluded studies should be provided.

6. Were the characteristics of the included studies provided? In an aggregated form such as a table, data from the original studies should be provided on the participants, interventions and outcomes. The ranges of characteristics in all the studies analyzed e.g. age, race, sex relevant socioeconomic data, disease status, duration, severity, or other diseases should be reported.

7. Was the scientific quality of the included studies assessed and documented? 'A priori' methods of assessment should be provided (e.g., for effectiveness studies if the author(s) chose to include only randomized, double-blind, placebo controlled studies or allocation concealment as includion criteria); for other types of studies alternative items will be relevant.

8. Was the scientific quality of the included studies used appropriately in formulating conclusions? The results of the methodological rigor and scientific quality should be considered in the analysis and the conclusions of the review, and explicitly stated in formulating recommendations.

9. Were the methods used to combine the findings of studies appropriate? For the pooled results, a test should be done to ensure the studies were combinable, to assess their homogeneity (i.e. Chi-squared test for homogeneity, 2). If heterogeneity exists a random effects model should be used and/or the clinical appropriateness of combining should be taken into consideration (i.e. is it sensible to combine?)

10. Was the likelihood of publication bias assessed? An assessment of publication bias should include a combination of graphical aids (e.g., funnel plot, other available tests) and/or statistical tests (e.g., Egger regression test).

11. Was the conflict of interest stated? Potential sources of support should be clearly acknowledged in both the systematic review and the included studies

\section{EVIDENCE OF BENEFIT}

\section{Strong Evidence}

There was no strong evidence to support or discount the use of any of the modalities in this overview.

\section{Moderate Evidence}

\section{Acupuncture}

We found acupuncture to be more beneficial relative to sham acupuncture (4 RCTs, 150 participants) [61-64] or sham laser or TENS (4 RCTs, 348 participants [65-68]) and a wait list or no treatment (1 RCT, 30 participants) [69] for pain reduction in the short term in patients with chronic mechanical neck disorder.

\section{Intermittent Traction}

Intermittent traction when compared to no treatment or placebo (2 RCTs, 173 participants) [70, 71] for chronic mechanical neck disorder, neck disorder with radiculopathy or degenerative changes showed reduced pain in the short term.

\section{LASER}

GaALAs $830 \mathrm{~nm}$ or $904 \mathrm{~nm}$ ) was shown to be better than placebo (5 RCTs, 286 participants) [72-76] for chronic neck pain, in reducing pain and improving function, global perceived effect and quality of life immediately post treatment and at intermediate (approximately six months) follow-up.

\section{Low or Very Low Evidence}

\section{Acupuncture}

Acupuncture was shown to be better than no treatment (1 RCT, 40 participants) [77] for chronic myofascial neck pain in the short term.
We found acupuncture to be more beneficial for chronic neck pain than massage (1 RCT, 177 participants) [65] immediate post treatment and in the short term and better than traction (2 RCTs, 589 participants) $[78,79]$ for global perceived effect immediately following treatment.

We found acupuncture to be more beneficial than multimodal physical therapy (details not reported) (1 RCT, number of participants not reported) [80] for radicular pain in the short term. We found acupuncture to be more beneficial than the injection of lidocaine ( $2 \mathrm{RCT}$, number of participants not reported) $[81,82]$ for non-specific neck pain in the short term.

\section{Ultrasound}

Continuous ultrasound was better than a control immediately post treatment and in the short term (2 RCTs, 150 participants) $[83,84]$ for myofascial pain. High-powered pain threshold with the probe placed over the trigger point and held motionless was more beneficial than conventional ultrasound (1 RCT, 60 participants) [84] for myofascial pain immediately post treatment.

\section{LASER}

HeNe-632.8nm with exercise (1 RCT, 60 participants) [85] was shown to be better than placebo for chronic myofascial pain immediately post treatment but not at intermediate follow-up of six months. GaAlAs-830nm or $904 \mathrm{~nm}$ (4 RCTs, 196 participants) [72, 74, 75] was better than placebo for subacute/chronic neck pain with associated osteoarthritis. 905nm-red (1 RCT, 60 participants) [86] was shown to be better than placebo for improving disability in acute neck pain with radiculopathy immediately following treatment. GaAs- $904 \mathrm{~nm}$ was better than placebo (1 RCT, 60 participants) [87] for reducing myofascial neck pain, improving function and quality of life immediately post treatment and in the short term. 
Table 3. Summary of Findings by GRADE (Quality of Evidence)

\begin{tabular}{|c|c|c|c|c|c|}
\hline \multirow[t]{2}{*}{$\mathbf{R x}$} & \multirow{2}{*}{$\begin{array}{l}\text { Treatment Details, Comparison } \\
\text { \& DISORDER TYPE }\end{array}$} & \multirow{2}{*}{$\begin{array}{c}\text { Primary Authors } \\
\text { (REVIEW Reference) }\end{array}$} & \multicolumn{3}{|c|}{ Quality of Evidence (GRADE*) } \\
\hline & & & Strong & Moderate & Low \\
\hline \multicolumn{6}{|c|}{ EVIDENCE of BENEFIT } \\
\hline $\begin{array}{c}\stackrel{0}{0} \\
\frac{e}{1 I}\end{array}$ & $\begin{array}{l}\text { TENS } v \text { s placebo } \\
\text { for WAD } \\
\text { CHRONIC MYOFASCIAL } \\
\text { Dosage range: } 14-20 \text { minutes, } 1 \text { or } 8-10 \text { sessions over } 2-5 \text { weeks }\end{array}$ & $\begin{array}{l}\text { b) Hsueh } 1997 \text { [38] } \\
\text { b) Smania } 2005 \text { [88] } \\
\text { a) Flynn } 1987 \\
\text { (GROSS } 2007 \text { [16], } \\
\text { KROELING 2009 [19], 2013 } \\
\text { [126], RICKARDS 2006 [17]) }\end{array}$ & & & $\begin{array}{l}\text { IP/ST pain, } \\
\text { function } \\
\text { and disability }\end{array}$ \\
\hline$\frac{\stackrel{\varrho}{0}}{\frac{\mathscr{e}}{I I}}$ & $\begin{array}{l}\text { TENS + another treatment } v s \text { that same treatment: } \\
\text { a) infrared, b) hotpack/exercise, } \\
\text { c) collar/exercise/analgesic } \\
\text { for a) CHRONIC b) MYOFASCIAL c) ACUTE } \\
\text { NECK PAIN } \\
\text { Dosage range: } 15-30 \text { minutes, } 1 \text { or } 12-19 \text { sessions over } 6 \text { weeks }\end{array}$ & $\begin{array}{l}\text { a) Chiu } 2005 \text { [108] } \\
\text { b) Hou } 2002[101] \\
\text { c) Nordemar } 1981[109] \\
\text { (KROELING } 2009 \text { [19], } 2013 \\
{[126] \text { ) }}\end{array}$ & & & $\begin{array}{l}\text { b) IP pain } \\
\text { c) ST pain } \\
\text { a) IT pain }\end{array}$ \\
\hline $\begin{array}{l}\stackrel{0}{0} \\
\frac{0}{I I}\end{array}$ & $\begin{array}{l}\text { EMS } v s \text { placebo } \\
\text { for CHRONIC MYOFASCIAL NECK } \\
\text { PAIN } \\
\text { Dosage: One } 20 \text { minute session }\end{array}$ & $\begin{array}{l}\text { Hsueh } 1997 \text { [38] } \\
\text { (KROELING 2009 [19], } 2013 \\
[126])\end{array}$ & & & IP pain \\
\hline $\begin{array}{l}\stackrel{0}{0} \\
\frac{\mathscr{e}}{I I}\end{array}$ & $\begin{array}{l}\text { Ultra-reiz vs standard physiotherapy (ice, home exercise, advice) } \\
\text { for ACUTE WAD } \\
\text { Dosage: } 15 \text { minutes, } 5 \text { sessions over } 1 \text { week }\end{array}$ & $\begin{array}{l}\text { Henriks } 1996 \text { [90] } \\
\text { (KROELING 2009 [19], 2013 } \\
\text { [126], VERHAGEN 2007 [13]) }\end{array}$ & & & $\begin{array}{l}\text { ST pain } \\
\text { (very low) }\end{array}$ \\
\hline $\begin{array}{l}\stackrel{0}{0} \\
\frac{\mathscr{Q}}{I I}\end{array}$ & $\begin{array}{l}\text { Pulsed Electromagnetic Field vs placebo (all studies) or standard } \\
\text { treatment [127] for CHRONIC OA } \\
\text { WAD } \\
\text { CHRONIC UNSPECIFIED NECK PAIN } \\
\text { Dosage range: } \\
30 \text { minutes, } 18 \text { sessions over } 4-6 \text { weeks, full body mat [128], } \\
2 x / \text { day for } 3 \text { weeks, technique not reported [129], } 16 \text { minutes local } \\
\text { magnet, } 8 \text { minutes full body mat } 2 x / \text { day for } 2 \text { weeks [127] } \\
\text { c) } 3 x \text { in } 3 \text { weeks, collar [130] }\end{array}$ & $\begin{array}{l}\text { Trock } 1994 \text { [129] } \\
\text { Sutbeyaz } 2006 \text { [128] } \\
\text { Thuile } 2002 \text { [127] } \\
\text { Foley-Nolan } 1990 \text { [131] } \\
\text { (CONLIN } 2005 \text { [132], } \\
\text { KROELING 2009 [19], } 2013 \\
\text { [126], VERHAGEN 2007 [13]) }\end{array}$ & & & $\begin{array}{l}\text { IP pain, GPE, } \\
\text { disability } \\
\text { ST pain } \\
\text { IP pain } \\
\text { IP pain }\end{array}$ \\
\hline$\frac{\stackrel{0}{0}}{\frac{\mathscr{Q}}{1}}$ & $\begin{array}{l}\text { Repetitive Magnetic Stimulation vs placebo } \\
\text { for CHRONIC MYOFASCIAL PAIN } \\
\text { Dosage: } 20 \text { minutes, } 10 \text { sessions over } 2 \text { weeks }\end{array}$ & $\begin{array}{l}\text { Smania } 2003 \text { [133] } \\
\text { (GROSS } 2007 \text { [16], } \\
\text { KROELING 2009 [19], } 2013 \\
\text { [126], RICKARDS 2006 [17]) }\end{array}$ & & & IP/ST pain \\
\hline 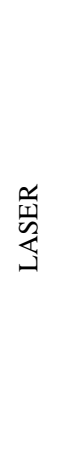 & $\begin{array}{l}\text { GaAlAs-830nm or 904nm vs placebo } \\
\text { for CHRONIC MND/DC (OA) } \\
\text { Dosage range: } 0.15 \text { to } 200 \text { seconds/point, } 10-14 \text { sessions, } 2-7 \\
\text { weeks } \\
\text { GaAs-904nm } v s \text { placebo } \\
\text { for CHRONIC MYOFASCIAL PAIN } \\
\text { Dosage: } 180 \text { seconds/point, } 10 \text { sessions over } 2 \text { weeks }\end{array}$ & $\begin{array}{l}\text { Ceccherelli } 1998 \text { [113] } \\
\text { Özdemer } 2001 \text { [74] } \\
\text { Taverna } 1990 \text { [76] } \\
\text { Soriano } 1996 \text { [75] } \\
\text { Chow } 2006 \text { [73] } \\
\text { Gur } 2004 \text { [87] } \\
\text { GROSS 2007 [16] and 2012 } \\
\text { [134], CHOW 2005 [135], } 2009 \\
\text { [136], LEAVER 2010 [137], } \\
\text { RICKARDS 2006 [17]) }\end{array}$ & & $\begin{array}{l}\text { IP/IT pain } \\
\text { IP/IT pain } \\
\text { IP function } \\
\text { IP/IT pain } \\
\text { IP/IT pain } \\
\text { IP pain, } \\
\text { function, } \\
\text { QoL, GPE }\end{array}$ & $\begin{array}{l}\text { IP, ST pain, } \\
\text { function, } \\
\text { QoL }\end{array}$ \\
\hline$\frac{\sqrt[c]{1}}{\sqrt[1]{4}}$ & $\begin{array}{l}\text { HeNe-632.8nm } v s \text { placebo for MYOFASCIAL PAIN } \\
\text { Dosage: irradiation time not reported, } \\
3 \text { sessions/week for } 4 \text { weeks }\end{array}$ & $\begin{array}{l}\text { Ibuldu } 2004 \text { [85] } \\
\text { GROSS } 2007 \text { [16] and } 2012 \\
\text { [134], CHOW 2009 [136], } \\
\text { RICKARDS 2006 [17]) }\end{array}$ & & & $\begin{array}{l}\text { IP pain, } \\
\text { physical } \\
\text { activity }\end{array}$ \\
\hline$\frac{\sqrt[c]{1}}{\sqrt[W]{4}}$ & $\begin{array}{l}905 \mathrm{~nm} \text { (red) } v s \text { placebo for ACUTE NECK PAIN WITH } \\
\text { RADICULOPATHY } \\
\text { Dosage: } 120 \text { seconds/point, } 5 \text { sessions/week for } 3 \text { weeks }\end{array}$ & $\begin{array}{l}\text { Konstantinovic } 2010 \text { [86] } \\
\text { (GROSS } 2012[134])\end{array}$ & & & $\begin{array}{l}\text { IP pain, } \\
\text { function, } \\
\text { QoL }\end{array}$ \\
\hline
\end{tabular}




\begin{tabular}{|c|c|c|c|c|c|}
\hline $\mathbf{R x}$ & $\begin{array}{l}\text { Treatment Details, Comparison } \\
\text { \& DISORDER TYPE }\end{array}$ & $\begin{array}{c}\text { Primary Authors } \\
\text { (REVIEW Reference) }\end{array}$ & \multicolumn{3}{|c|}{ Quality of Evidence (GRADE*) } \\
\hline \multicolumn{6}{|c|}{ EVIDENCE of BENEFIT } \\
\hline 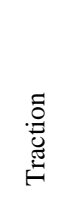 & $\begin{array}{l}\text { Intermittent Traction } v s \text { a) no treatment } \mathrm{b} \text { ) placebo } \\
\text { for CHRONIC MND, NDR, DC } \\
\text { Dosage: a) not reported b) } 10 \text { seconds on/off, } 15 \text { minutes, 2x/week } \\
\text { for } 6 \text { weeks }\end{array}$ & $\begin{array}{l}\text { a) Goldie 1970(62) } \\
\text { b)Zylbergold } 1985 \text { [71] } \\
\text { (GRAHAM } 2006 \text { [21] and } 2008 \\
\text { [138], GROSS } 2007 \text { [16], KAY } \\
2009 \text { [139]) }\end{array}$ & & $\begin{array}{l}\text { a) ST GPE } \\
\text { b) ST pain }\end{array}$ & \\
\hline 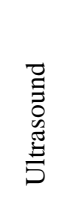 & $\begin{array}{l}\text { Continuous Ultrasound } v s \text { active control (stretching) for } \\
\text { MYOFASCIAL NECK PAIN } \\
\text { Dosage: a) } 1-5 \text { sessions over } 4 \text { weeks } \\
\text { b) } 10 \text { sessions over } 2 \text { weeks }\end{array}$ & $\begin{array}{l}\text { a) Maljesi 2004(76) } \\
\text { b) Esenyel } 2000 \text { [83] } \\
\text { (GRAHAM } 2008 \text { [140], GROSS } \\
\text { 2007 [16], PELOSO 2007 [141], } \\
\text { RICKARDS } 2006 \text { [17]) }\end{array}$ & & & $\begin{array}{l}\text { IP/ST pain } \\
\text { IP pain }\end{array}$ \\
\hline 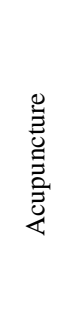 & $\begin{array}{l}\text { Acupuncture } v s \text { sham (LASER, TENS) for } \\
\text { CHRONIC NECK PAIN } \\
\text { TCM approach, dry needles } \\
\text { Standard points } \\
\text { TCM approach } \\
\text { Western approach } \\
\text { Dosage range: } 20-30 \text { minutes, } 1 \text { to } 8 \text { sessions over } \\
\text { 3-4 weeks }\end{array}$ & $\begin{array}{l}\text { Irnich } 2002 \text { [66] } \\
\text { Petrie } 1983 \text { [67] } \\
\text { Irnich } 2001 \text { [65] } \\
\text { White } 2004 \text { [145] } \\
\text { (FU 2009 [143], TRINH } 2006 \\
\text { [144] and 2007 [27], GROSS } \\
2007 \text { [16], HARALDSSON } \\
2006[146] \text { ) }\end{array}$ & & ST pain & \\
\hline 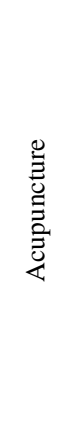 & $\begin{array}{l}\text { Acupuncture } v s \text { comparison treatment a) massage b) and c) } \\
\text { traction for CHRONIC NECK PAIN } \\
\text { a) and b) TCM approach } \\
\text { c) electro-acupuncture } \\
\text { Dosage: } 30 \text { minutes, } 5 \text { sessions over } 3 \text { weeks } \\
\text { Dosage: } 1 \text { session every other day, } 7 \text { in total, timing not reported } \\
\text { [79], } 30-40 \text { minutes, } 3 \text { sessions/week for } 6 \text { weeks [78] }\end{array}$ & $\begin{array}{l}\text { Irnich } 2001 \text { [65] } \\
\text { (FU 2009 [143], FURLAN } 2012 \\
\text { [147], TRINH 2006 [144] and } \\
2007 \text { [27], HARALDSSON } \\
2006 \text { [145]) } \\
\text { b) Guangyue 2001 [79] } \\
\text { c) Loy } 1983 \text { [78] } \\
\text { (GRAHAM 2006 [21], TRINH } \\
\text { 2006 [144] and 2007 [27]) }\end{array}$ & & & $\begin{array}{l}\text { a) IP, ST pain } \\
\text { b) and c) IP, } \\
\text { GPE }\end{array}$ \\
\hline 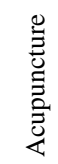 & $\begin{array}{l}\text { Acupuncture } v s \text { comparison treatment (physical therapy for } \\
\text { RADICULOPATHY } \\
\text {-Traditional Chinese Medicine approach } \\
\text { Dosage: not reported }\end{array}$ & $\begin{array}{l}\text { Zhou } 2006[148] \\
\text { (FU } 2009[143])\end{array}$ & & & ST pain \\
\hline 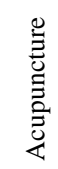 & $\begin{array}{l}\text { Acupuncture } v s \text { lidocaine injection for NECK PAIN } \\
\text {-Traditional Chinese Medicine approach } \\
\text { Dosage: not reported }\end{array}$ & $\begin{array}{l}\text { Fu } 2005[81] \\
\text { Wang } 2007[82] \\
\text { (FURLAN } 2012[147])\end{array}$ & & & ST pain \\
\hline
\end{tabular}


(Table 3) contd......

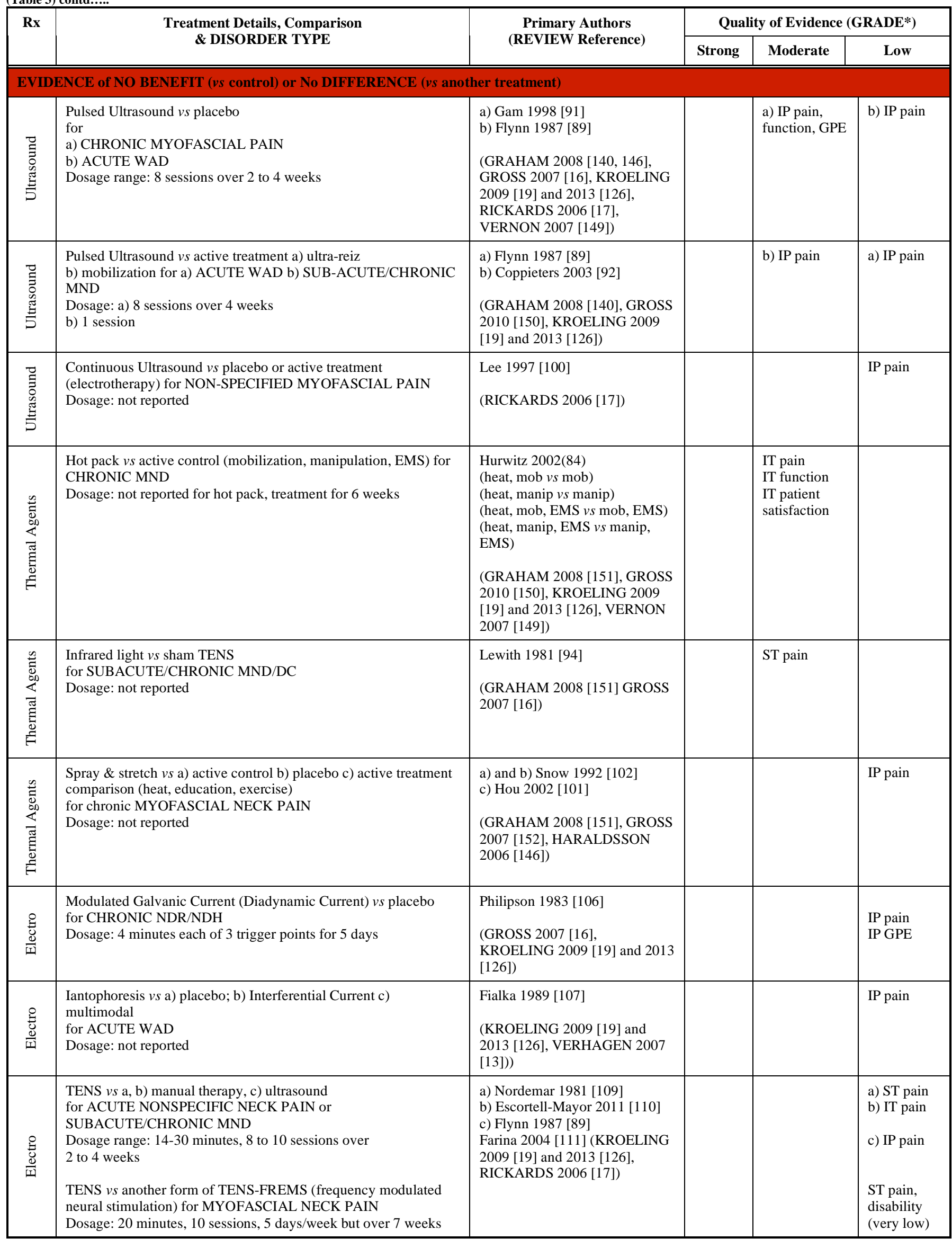




\begin{tabular}{|c|c|c|c|c|c|}
\hline \multirow[t]{2}{*}{$\mathbf{R x}$} & \multirow{2}{*}{$\begin{array}{l}\text { Treatment Details, Comparison } \\
\text { \& DISORDER TYPE }\end{array}$} & \multirow{2}{*}{$\begin{array}{c}\text { Primary Authors } \\
\text { (REVIEW Reference) }\end{array}$} & \multicolumn{3}{|c|}{ Quality of Evidence (GRADE*) } \\
\hline & & & Strong & Moderate & Low \\
\hline \multicolumn{6}{|c|}{ EVIDENCE of NO BENEFIT ( $v$ s control) or No DIFFERENCE ( $v s$ another treatment) } \\
\hline$\frac{\stackrel{0}{0}}{\stackrel{\varrho}{\Psi}}$ & $\begin{array}{l}\text { Magnetic Necklace (Static Magnet) vs placebo } \\
\text { for chronic MND } \\
\text { Dosage: } 24 \text { hours daily for } 3 \text { weeks }\end{array}$ & $\begin{array}{l}\text { Hong } 1982[153] \\
\text { (GROSS } 2007[16], \\
\text { KROELING 2009 [19] and } 2013 \\
[126])\end{array}$ & & & IP pain \\
\hline $\begin{array}{l}\stackrel{\Xi}{0} \\
\frac{\mathscr{Q}}{I I}\end{array}$ & $\begin{array}{l}\text { EMS } v s \text { EMS + other treatment (mobilization or manipulation, } \\
\text { heat) for SUBACUTE/ } \\
\text { CHRONIC MND, NDR, NDH } \\
\text { Dosage: unclear } \\
\text { EMS for CHRONIC MYOFASCIAL PAIN } v s \text { TENS } \\
\text { Dosage: } 1 \text { session for } 20 \text { minutes }\end{array}$ & $\begin{array}{l}\text { Hurwitz } 2002 \text { [93] } \\
\text { (EMS, manip } v s \text { manip) } \\
\text { (EMS, mob vs mob) } \\
\text { (EMS, heat, manip } v s \text { heat, } \\
\text { manip) } \\
\text { (EMS, heat, mob } v \text { s heat, mob) } \\
\text { Hseuh } 1997 \text { [38] } \\
\text { (GRAHAM } 2008 \text { [151], GROSS } \\
\text { 2007 [16] and } 2010 \text { [150, 146], } \\
\text { KROELING } 2009 \text { [19], 2013 } \\
\text { [126], RICKARDS 2006 [17]) }\end{array}$ & & & $\begin{array}{l}\text { IT pain, } \\
\text { function } \\
\text { ST patient } \\
\text { satisfaction } \\
\text { IP pain }\end{array}$ \\
\hline $\begin{array}{l}\stackrel{\ominus}{0} \\
\frac{\mathscr{e}}{I I}\end{array}$ & $\begin{array}{l}\text { Pulsed Electromagnetic Field } v s \text { placebo for CHRONIC NECK } \\
\text { PAIN } \\
\text { Dosage: } 8 \text { hours daily for } 12 \text { weeks }\end{array}$ & $\begin{array}{l}\text { Foley-Nolan } 1992[130] \\
\text { (KROELING 2009 [19] and } \\
2013 \text { [126], VERHAGEN } 2007 \\
[13])\end{array}$ & & & IP pain \\
\hline $\begin{array}{c}\stackrel{0}{0} \\
\frac{\mathscr{Q}}{I I}\end{array}$ & $\begin{array}{l}\text { TENS vs manipulation for CHRONIC CERVICOGENIC } \\
\text { HEADACHE WITH DEGENERATIVE CHANGE } \\
\text { Dosage: } 20 \text { minutes every other day for } 10 \text { sessions } \\
\text { vs exercise, infrared for CHRONIC NON-SPECIFIC NECK PAIN } \\
\text { Dosage: } 30 \text { minutes, } 2 \text { sessions over } 6 \text { weeks }\end{array}$ & $\begin{array}{l}\text { Chen } 2007[154] \\
\text { (GROSS } 2010[150]) \\
\text { Chiu } 2005[108] \\
\text { (KROELING } 2009[19] \text { and } \\
2013[126])\end{array}$ & & & $\begin{array}{l}\text { ST pain } \\
\text { (very low) } \\
\text { IT pain } \\
\text { IT function } \\
\text { (very low) }\end{array}$ \\
\hline 密 & $\begin{array}{l}\text { GaAs-830nm } v s \text { placebo } \\
\text { for SUBACUTE/CHRONIC MND (MYOFASCIAL) } \\
\text { Dosage: } 180 \text { seconds/point } 3 \text { sessions/week for } 2 \text { weeks } \\
\text { for CHRONIC MND (myofascial) } \\
\text { Dosage range: } 30-60 \text { seconds/point, } 6 \text { to } 14 \text { sessions over } 2 \text { to } 7 \\
\text { weeks }\end{array}$ & $\begin{array}{l}\text { Thorsen } 1991 \text { [112] } \\
\text { Chow } 2004 \\
\text { Thorsen } 1992 \text { [114] } \\
\text { Seidel } 2002 \text { [105] } \\
\text { (CHOW 2009 [134], GROSS } \\
\text { 2007 [16] and 2012 [134], } \\
\text { LEAVER } 2010 \text { [137]) }\end{array}$ & & $\begin{array}{l}\text { ST pain } \\
\text { ST pain } \\
\text { ST pain }\end{array}$ & $\begin{array}{l}\text { IP pain, } \\
\text { QoL, } \\
\text { GPE }\end{array}$ \\
\hline 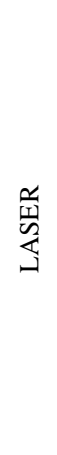 & $\begin{array}{l}\text { HeNe-632.8nm vs placebo } \\
\text { for CHRONIC MND (myalgia) } \\
\text { Dosage: } 15 \text { seconds/point } 2 \text { sets of } 5 \text { daily consecutive sessions } \\
\text { with } 6 \text { week break between } \\
\text { for MYOFASCIAL PAIN } \\
\text { Dosage: timing for each point not reported, } 3 \text { sessions over } 4 \\
\text { weeks } \\
\text { for ACUTE WAD } \\
\text { Dosage: only reports treatment for } 3 \text { weeks }\end{array}$ & $\begin{array}{l}\text { Waylonis } 1988 \text { [155] } \\
\text { Ilbuldu } 2004 \text { [85] } \\
\text { (CHOW } 2009 \text { [136], GROSS } \\
2007 \text { [16] and 2012 [134]) } \\
\text { Aigner 2006 [156] } \\
\text { (CHOW 2009 [136], } \\
\text { VERHAGEN 2007 [13]) }\end{array}$ & & & $\begin{array}{l}\text { IT pain, } \\
\text { physical } \\
\text { function } \\
\text { IP/IT pain }\end{array}$ \\
\hline 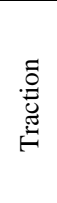 & $\begin{array}{l}\text { Continuous Traction } v \text { s placebo } \\
\text { for ACUTE TO CHRONIC MND, NDR, DC } \\
\text { Dosage range: } 15-30 \text { minutes, } 12 \text { sessions over } 4 \text { to } 6 \text { weeks }\end{array}$ & $\begin{array}{l}\text { Brewerton 1966(63) } \\
\text { Klaber Moffett } 2006[96] \\
\text { Zylbergold } 1985[71] \\
\text { (GRAHAM } 2006 \text { [21] and } 2008 \\
\text { [138]) }\end{array}$ & & $\begin{array}{l}\text { ST pain } \\
\text { ST function }\end{array}$ & \\
\hline
\end{tabular}


(Table 3) contd.....

\begin{tabular}{|c|c|c|c|c|c|}
\hline \multicolumn{6}{|c|}{ EVIDENCE of NO BENEFIT ( $v$ s control) or No DIFFERENCE ( $v$ s another treatment) } \\
\hline 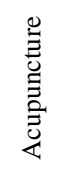 & $\begin{array}{l}\text { Acupuncture } v s \text { comparison treatment (physical therapy) for } \\
\text { CHRONIC NECK PAIN } \\
\text { - local trigger points } \\
\text { Dosage: } 15 \text { minutes, } 6 \text { sessions over } 1 \text { week }\end{array}$ & $\begin{array}{l}\text { David } 1998 \text { [157] } \\
\text { (GROSS } 2010 \text { [149], TRINH } \\
2006[144] \text { and 2007 [27]) }\end{array}$ & & & $\begin{array}{l}\text { ST pain } \\
\text { (very low) }\end{array}$ \\
\hline 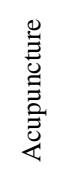 & $\begin{array}{l}\text { Electro-acupuncture } v s \text { a) oral tenoxicam (NSAID) with ranitidine } \\
\text { b) manipulation for CHRONIC MND/DC } \\
\text { Dosage: timing not reported, } 6 \text { sessions over 3-4 weeks }\end{array}$ & $\begin{array}{l}\text { Giles } 1999 \text { [97] } \\
\text { (GROSS 2010 [150], PELOSO } \\
2007[153])\end{array}$ & & & $\begin{array}{l}\text { IP pain } \\
\text { IP function }\end{array}$ \\
\hline 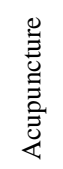 & $\begin{array}{l}\text { Acupuncture } v s \text { oral diazepam for CHRONIC MND/OA } \\
\text { Dosage: one } 40 \text { minute session }\end{array}$ & $\begin{array}{l}\text { Thomas } 1991[98] \\
\text { (PELOSO } 2007[141])\end{array}$ & & & IP pain \\
\hline
\end{tabular}

Key: GRADE* : study design, within study risk of bias, consistency of results, directness (generalizability), precision (sufficient data), reporting bias (publication, language, funding, other); WAD - whiplash associated disorder; MND - mechanical neck disorder; DC - degenerative change; OA - osteoarthritis; NDR - neck disorder with radiculopathy; NDH neck disorder with headache; TENS - Transcutaneous Electrical Nerve Stimulation; EMS - electrical muscle stimulation; TCM - Traditional Chinese Medicine; NDI - neck disability index; QoL - quality of life; GPE - global perceived effect; IP - immediate post treatment; ST - short term closest to 3 months, IT - intermediate term closest to 6 months, $\mathrm{LT}$ - long term closest to 1 year; $v s$ - versus; nm - nanometer

\section{Electrotherapy}

\section{TENS}

TENS (3 RCTs, 88 participants) [38, 88, 89] was more beneficial for pain reduction when compared to placebo for myofascial pain or WAD of unspecified duration immediately following treatment. Ultra-Reiz, a form of TENS (1 RCT, 16 participants) [90] was more beneficial for reducing acute WAD pain when compared to standard physiotherapy including ice, home exercise and advice in the short term. TENS was beneficial for reducing chronic myofascial neck pain (1 RCT, 60 participants) [38] when compared to electric muscle stimulation immediately post treatment.

\section{EVIDENCE OF NO BENEFIT (VS CONTROL) OR NO DIFFERENCE (VS ANOTHER TREATMENT)}

\section{Moderate Evidence}

\section{Ultrasound}

Pulsed ultrasound was no better than placebo (2 RCT, 79 participants) [89, 91] at changing function or global perceived effect immediately post treatment in patients with either acute WAD or chronic myofascial neck pain. Also, ultrasound was inferior to mobilization (1 RCT, 20 participants) [92] for subacute/chronic neck pain immediately post treatment.

\section{Thermal Agents}

There was no difference between hot packs (1 RCT, 269 participants) [93] and an active control (mobilization, manipulation or EMS) at improving pain, function and patient satisfaction in the intermediate term for patients with chronic mechanical neck disorder. There was no difference when infrared light was compared to sham TENS (1 RCT, 26 participants) [94] for subacute/chronic neck pain in the short term.

\section{Continuous Traction}

There was no difference when continuous traction was compared to placebo (3 RCTs, 606 participants) [71, 95, 96] for improving pain or function in patients with acute to chronic neck pain in the short term.

\section{Low or Very Low Evidence}

\section{Acupuncture}

There was no difference found when electro (1 RCT, 62 participants) [97] or non-stimulated acupuncture (1 RCT, 132 participants) [98] for chronic mechanical neck disorder

Table 4. Therapies with Conflicting Evidence

\begin{tabular}{|c|c|}
\hline Treatments with Conflicting Evidence & Author \\
\hline \hline $\begin{array}{c}\text { LASER-904nm, 830nm, 780nm vs control } \\
\text { for CHRONIC MYOFASCIAL PAIN }\end{array}$ & (GROSS 2007 [16], 2012 [134] and CHOW 2005 [135], CHOW 2009 [136], LEAVER 2010 [137]) \\
\hline
\end{tabular}


Table 5. Evidence-Based Recommendations

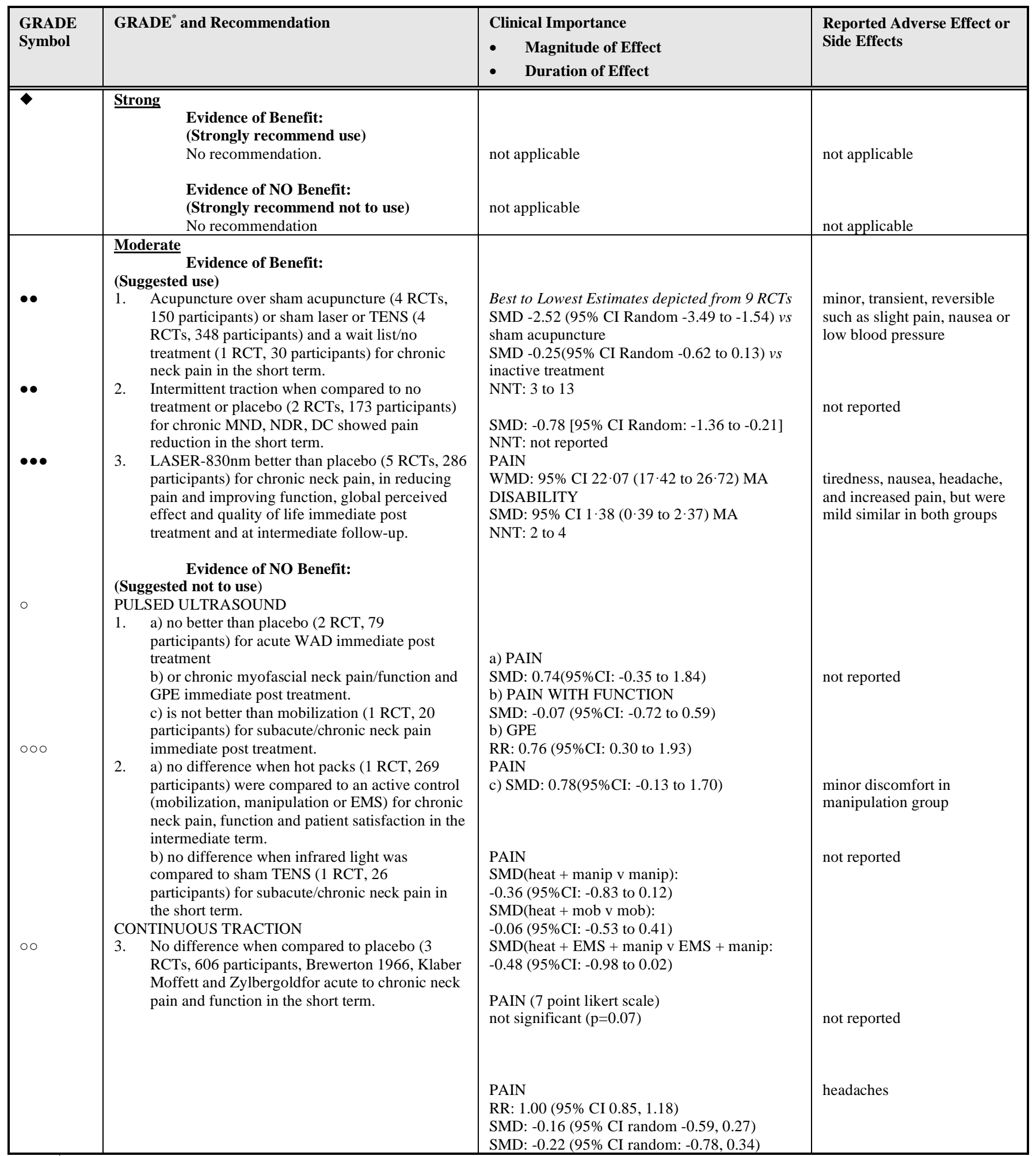

GRADE*: study design, within study risk of bias, consistency of results, directness (generalizability), precision (sufficient data), reporting bias (publication, language, funding, other); open symbol= no benefit; closed symbol = beneficial; duration of effect noted by number of symbols: one $=$ IP, two $=$ ST, three $=$ IT, $4=$ LT; diamond $(\bullet)=$ high GRADE; $\operatorname{dot}(\bullet)=$ moderate GRADE.

Clinically Important is determined by considering the following factors: minimal detectable change, minimal clinically important difference ( $\geq 15 \%)$, large magnitude of effect (weighted mean difference, number needed to treat, absolute benefit, treatment advantage), high dose response gradient, duration of the effect (IP - immediate post treatment, ST short term for about 3 months, IT - intermediate term for about 6 months, LT - long term for about 1 year).

Key: WAD - whiplash associated disorder; MND - mechanical neck disorder; SMDp - Standard Mean Difference pooled; WMDp - weighted mean difference pooled; RR - relative risk; NNT - number needed to treat; $95 \%$ CI - 95\% confidence interval, GPE - global perceived effect. 


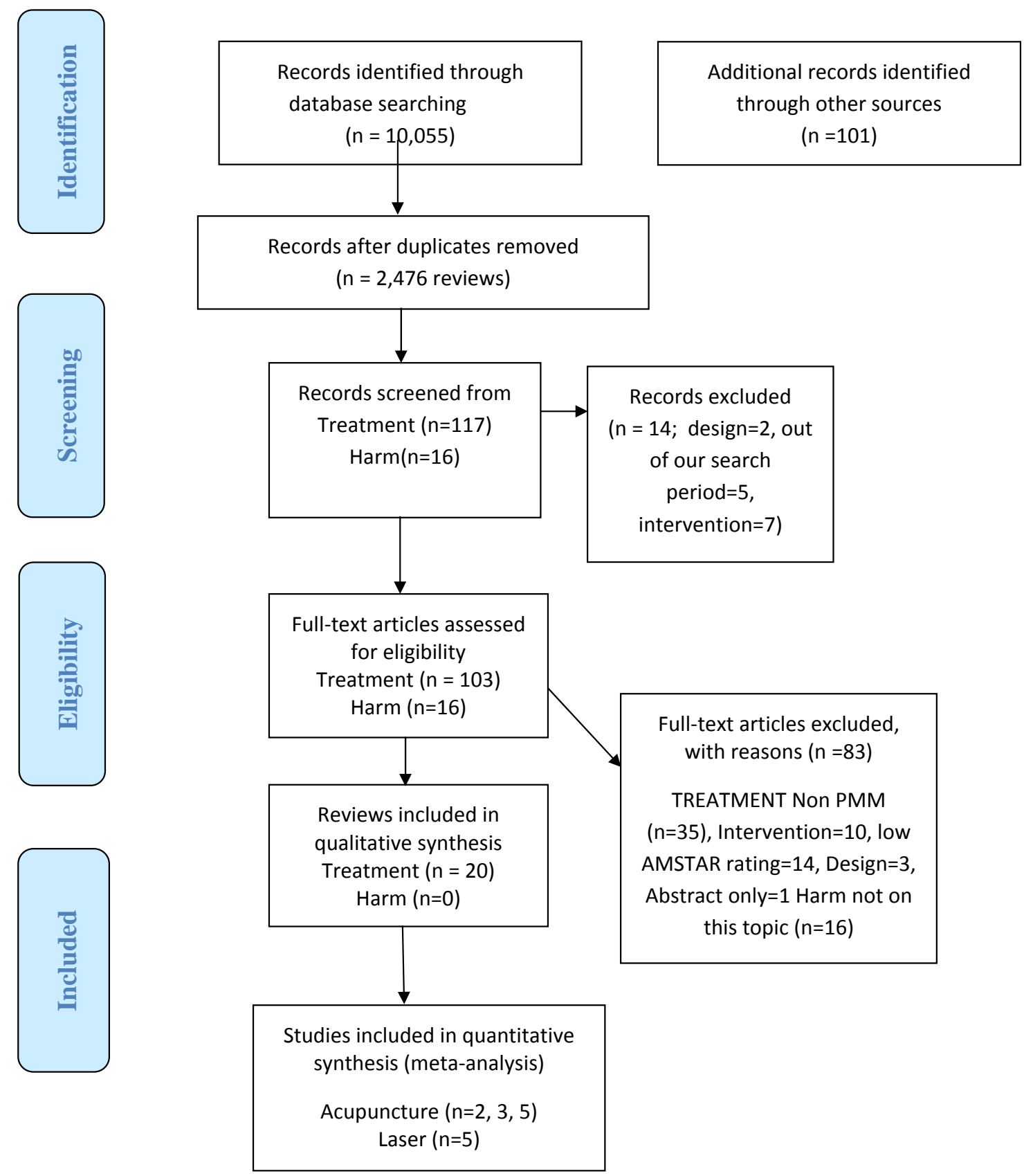

Fig. (1). PRISMA diagram showing the flow of reviews for Physical Medicine Methods (PMM).

with degenerative changes was compared to manipulation or medication-tenoxicam (NSAID) with ranitidine or diazepam respectively for improving pain or function immediately following treatment. Additionally, no difference was found between acupuncture and manipulation for chronic neck pain (1 RCT, 69 participants) [99] at long term follow-up.

\section{Ultrasound}

Pulsed ultrasound was inferior to ultra-reiz (1 RCT, 21 participants) [89] for acute WAD immediately post treatment. Continuous ultrasound was not beneficial when compared to placebo and inferior to an active treatment (electrotherapy, type not specified) (1 RCT, 26 participants)
[100] for reducing myofascial neck pain immediately post treatment.

\section{Thermal Agents}

No difference was found when spray/stretch was compared to an active control, placebo or active treatment (heat, education, exercise) (2 RCTs, 72 participants) [101, 102] for reducing chronic myofascial neck pain immediately post treatment.

\section{LASER}

For Helium Neon 632.8nm (1 RCT, 55 participants) [103] there was no benefit for pain relief when compared to placebo immediately following treatment for chronic 
myofascial pain syndrome. For pain reduction, GaAs 830nm was inferior to manipulation (1 RCT, 38 participants) [104] for cervicogenic headache or acupuncture (1 RCT, 12 participants) [105] for chronic tendomyositis both in the short term.

\section{Electrotherapy}

\section{Modulated Galvanic Current}

There was no benefit for improving chronic neck pain and global perceived effect (1 RCT, 40 participants) [106] compared to placebo immediately post treatment.

\section{Iontophoresis}

There was no benefit when iontophoresis was compared to no treatment, interferential current and was inferior to a multimodal approach of traction, exercise and massage (1 RCT-3 arms) [107] for improving pain in patients with acute WAD with cervicogenic headache immediate post treatment.

\section{TENS}

There was no benefit when TENS was compared to placebo (1 RCTs, 53 participants) [88] for reducing pain in patients with chronic mechanical neck disorder immediately post treatment.

There was no benefit adding TENS to other treatments (infrared, hotpack/exercise, collar/exercise/analgesic, and standard physiotherapy of ice/home exercise/advice) [90, $101,108,109]$ for various disorder types and various followup periods in very small trials. There was no difference when TENS was compared to manual therapy (2 RCTs, 107 participants) [109, 110] or ultrasound (1 RCT, 14 participants) [89] for reducing subacute/chronic neck pain immediately post treatment. Also, when different parameters of TENS were compared to each other the results were similar for pain at short term follow-up (1 RCT, 40 participants) [111].

\section{CONFLICTING EVIDENCE}

\section{Laser}

$830 \mathrm{~nm}$ or $904 \mathrm{~nm}$ for myofascial pain had varied evidence (5 RCTs, Ceccherelli 1989, Gur 2004, Seidel 2002, Thorsen 1991, Thorsen 1992) [87, 105, 112-114] with metaregression (2 RCTs, 81 participants, Ceccherelli 1989 and Gur 2004) [87, 113] suggesting drive technology (superpulse) may be most successful in treating this subgroup albeit an independent clinical trial is needed to demonstrate this. We have also recorded trials with conflicting/unclear evidence in Table 3 .

\section{ADVERSE EVENTS}

We found minor, transient, and reversible side effects consisting of increased pain, headache, tiredness or nausea to be occasionally present when reported for physical modalities. Most trials did not report adverse events at all or if so, collection of data was not described adequately.

\section{DISCUSSION}

Although the evidence continues to improve with respect to the use or not of physical modalities as a treatment choice for management of neck pain, there is still a lack of strong evidence to base recommendations that address meaningful outcomes in a standardized way. The core recommendations based on moderate evidence may be strengthened or refuted by results from larger trials with sound methodological quality. These recommendations supporting the use of acupuncture, laser and intermittent traction but not pulsed ultrasound, hot packs and continuous traction are summarized in Table $\mathbf{5}$.

Some modalities especially acupuncture and laser, utilize a broad spectrum of doses that may be beneficial while others appear to be of no benefit for pain management. Specific dosage trials are essential. Data on function/disability and quality of life is limited or completely absent. Many studies focus on pain as the primary outcome and to a lesser extent function typically using differing outcomes. Unless there is consistent use of the same pain and disability outcomes in these clinical trials it will be difficult to undertake meta-analyses and provide clinicians with a summary estimate of the expected benefit when considering these two outcomes. Further, since physical agents can be used for a variety of adjunctive benefits such as enhancing tissue extensibility to improve motion gains during mobilizations or exercise; or general healing effects then short term pain measures may not capture their benefit.

A number of reporting and design issues are consistent across neck clinical trials and have been detailed in Goldsmith et al. 2011 [115]. However, there are design options and clear criteria that can be used to improve the feasibility and quality of future clinical trials in this area of neck pain but also in rehabilitation generally. In particular, future research should ensure adherence to reporting standards of CONSORT [116, 117] and PRISMA guidelines, and should look beyond the basic two group design commonly used in clinical trials as well as consistent use of similar impairment and disability outcomes. Since rehabilitation often includes multiple interventions, it is recommended that researchers use factorial design methods to not only evaluate the effectiveness of independent treatments but also the impact on various combinations within the same trial. A core set of patient reported outcomes and key participation indicators (such as return to work) are needed and the community at large should consider setting initiatives, similar to the International Classification of Functioning (ICF) core measures [118]. Additionally, most studies in this overview assessed the outcome immediately following treatment up to six months. There is a need for more long-term follow-up.

There is general agreement between reviews. Some of the primary studies in one review may differ from another due to inclusion/exclusion criteria or search strategies.

Consistent reporting of side effects and how this information was collected is poor in the majority of trials. Accurate prospective collection of adverse event data is fundamental to these trials; there has been work done to define categories of severity and types of events considered adverse within the musculoskeletal area [119-121]. A move toward more active methods of capturing harms (rather than spontaneous reporting) would be necessary. Although our search did not retrieve any reviews of harms for physical modalities specific to neck pain, we can look to reviews pertaining to other musculoskeletal conditions or the general use of some physical modalities such as acupuncture. A 
review on the safety of acupuncture was reported by Wheway et al. [122] after analyzing the National Reporting and Learning Database of the National Patient Safety Agency in the United Kingdom. The authors reported the following adverse events related to 325 patient incidents: retained needles (31\%), dizziness (30\%), loss of consciousness/unresponsive (19\%), falls (4\%), bruising or soreness at needle site (2\%), Pneumothorax (1\%) and other adverse reactions $(12 \%)$. More than $95 \%$ of the incidents were categorized as low or no harm. Other reviews have reported more serious adverse events related to acupuncture [123] however these findings should be questioned due to the use of unclear and unsystematic review methods that may have produced biased results. As it is well known that acupuncture treatment can be similar in technique regardless of the condition treated, it is conceivable that the aforementioned adverse events could manifest in patients with neck pain.

In a systematic review of conservative treatments for lumbar disc herniation, traction was related to 16 adverse events ranging from increased pain, anxiety, and fainting to lower limb weakness [124]. As there are many differences in the application of traction to the neck and low back, these specific results may not be directly relevant. While these adverse events appear to be minor and transient, they are perhaps an indication of the susceptibility of the spine when forces are applied in less specific and less controlled ways i.e. external machines, than with the sensitivity afforded by manual techniques. A physical modality such as hot packs for example, lacks strong evidence of benefit but harm is minimal and may still be considered since they are cost effective, easy to apply in a self-management program and may provide an element of comfort to the patient. Even though supportive evidence is lacking, it is only one component of evidence-based practice to be considered in the development of an individual treatment plan but decision making also requires the therapist's clinical experience and the patient's wishes [125].

We had several strengths regarding our approach to summarizing this literature. We used a comprehensive, librarian-assisted search and multiple databases. Two independent reviewers determined article relevance, assessed the AMSTAR methodological and verified data extraction. Consensus was used both by the internal data abstraction team and an interdisciplinary external panel consensus to validate the GRADE of evidence and recommendations.

\section{CONCLUSION}

\section{Final Evidence-Based Recommendations (Table 4)}

For chronic mechanical neck disorders, the recommendation from moderate evidence suggests using acupuncture or intermittent traction for short term pain reduction. In addition, this evidence suggests using LASER $830 \mathrm{~nm}$ and $904 \mathrm{~nm}$ for pain reduction, improving function/quality of life and to maximize global perceived effect up to the intermediate term (approximately six months). For both acute WAD and subacute/chronic neck disorders (including myofascial pain), the evidence suggests there is no benefit using pulsed ultrasound for reducing pain, improving function or global perceived effect immediately following treatment. Thermal agents (hot pack and infrared light) for chronic neck pain immediately following treatment or in the short term are also not suggested. Lastly, the evidence suggests there is no benefit in continuous traction for acute to chronic neck disorders for short term pain relief and improved function. Some electrotherapies (magnetic necklace, galvanic current) may not be beneficial for chronic neck pain. Dosage, design and long term follow-up continue to be the recommendations and essential elements when designing future trials.

\section{CONFLICT OF INTEREST}

The authors confirm that this article content has no conflict of interest.

\section{ACKNOWLEDGEMENTS}

This work was supported by Canadian Institutes of Health Research (CIHR) grant(s) FRN: KRS-102084 and Centric Health LifeMark Industry Partner Grant.

\section{APPENDIX 1}

Excluded Studies Categorized by Reason for Exclusion

Excluded for Laser

\section{Design}

Tsakitzidis G, Remmen R, Peremans L, Van Royen P, Duchesnes C, Paulus D, et al. Non-specific neck pain: diagnosis and treatment. Good Clinical Practice (GCP). Brussels: Belgian Health Care Knowledge Centre (KCE) 2009; Report No.: KCE Reports 119C.

\section{Low AMSTAR rating}

Brønfort G, Haas M, Evans R, Leininger B, Triano J. Effectiveness of manual therapies: The UK evidence report. Chiropractic and Osteopathy 2010; 18: 3.

Hurwitz EL, Carragee EJ, van der Velde G, Carroll LJ, Nordin M, Guzman J etal. Treatment of neck pain; noninvasive interventions: results of the Bone and Joint Decade 2000-2010 task force on neck pain and its assoiciated disorders. Spine 2008; 33(4 Suppl); S123-S152.

Fernandez-de-Las PC, Alonso-Blanco C, Cuadrado ML, Pareja JA. Spinal Manipulative Therapy in the Management of Cervicogenic Headache. Headache 2005; 45: 1260-70.

Teasell RW, McClure JA, Walton D, Pretty J, Salter K, etal. A research synthesis of therapeutic interventions for whiplash-associated disorders (WAD): Part 2-Interventions for WAD. Pain Res Manage 2010; 15(5): 295-304.

Vernon H, Schneider M. Chiropractic management of myofascial trigger points and myofascial pain syndrome: A systematic review of the literature [review] J Manipulative Physiol Ther 2009; 32(1): 14-24.

Excluded for Acupuncture

\section{Intervention}

Conlin A, Bhogal S, Sequeira A, Teasell R. Treatment of whiplash-associated disorders-Part 1: Non-invasive interventions. Pain Res Manage 2005; 10(1): 21-32. 
Haines T, Gross A, Burnie SJ, Goldsmith CH, Perry L. Patient education for neck pain with or without radiculopathy. [Review] [123 refs][Update of Cochrane Database Syst Rev 2008; (4): CD005106; PMID: 18843681]. Cochrane Database Syst Rev 2009; (1): CD005106.

Miller J, Gross A, D'Sylva J, Burnie SJ, et al. Manual therapy and exercise for neck pain: A systematic review Man Ther 2010; 15: 334-54.

Wang M-Y, Tsai P-S, Lee P-H, Chang W-Y, Yang C-M. Systematic review and meta-analysis of the efficacy of tuina for cervical spondylosis. J Clin Nurs 2008; 17: 2531-8.

\section{Low AMSTAR Rating}

Baxter GD, Bleakley C, McDonough S. Clinical effectiveness of laser acupuncture: A systematic review. JAMS J Acupunct Meridian Stud 2008; 1(2): 65-82.

Brønfort G, Haas M, Evans R, Leininger B, Triano J. Effectiveness of manual therapies: The UK evidence report. Chiropr Osteopat 2010; 18: 3.

Hurwitz EL, Carragee EJ, van der Velde G, et al. Treatment of neck pain; noninvasive interventions: results of the Bone and Joint Decade 2000-2010 Task Force on Neck Pain and Its Assoiciated Disorders. Spine 2008; 33(4 Suppl); S123-S152.

Lee MS, Choi T-Y, Kim J-I, Choi S-M. Using Guasha to treat musculoskeletal pain: A systematic review of controlled clinical trials. Chinese Med 2010; 5: 5.

Lin H-L, Song H-M, Zhong W-H, Chen S-Q, Wang S-Z. Effects of different acupuncture and moxibustion treatments on cervical spondylotic radiculopathy: A systematic evaluation. [Chinese]. J Clin Rehab Tissue Eng Res 2009; 13(46): 9017-21.

Reid SA, Rivett DA. Manual therapy treatment of cervicogenic dizziness: A systematic review. Manual Ther 2005: 4-13.

Vernon H, Humphreys BK. Manual therapy for neck pain: an overview of randomized clinical trials and systematic reviews. [Review] [141 refs]. Europa Medicophysica 2007; 43(1): 91-118.

Vernon H, Schneider M. Chiropractic management of myofascial trigger points and myofascial pain syndrome: A systematic review of the literature [review] J Manipulative Physiol Ther 2009; 32(1): 14-24.

\section{Design}

Ernst E, Lee MS, Choi TY. Acupuncture: does it alleviate pain and are there serious risks? A review of reviews. Pain 2011; 152(4): 755-64.

Itoh K, Kitakoji H. Acupuncture for chronic pain in Japan: A review. Evidence-based Complementary and Alternative Medicine 2007; 4(4): 431-8. [neck studies included were non-RCTs]

Tsakitzidis G, Remmen R, Peremans L, Van Royen P, Duchesnes C, Paulus D, et al. Non-specific neck pain: diagnosis and treatment. Good Clinical Practice (GCP). Brussels: Belgian Health Care Knowledge Centre (KCE) 2009; Report No.: KCE Reports 119C.
Wang YW, Fu WB, Ou, AH, Fan L, Huang YF. A systematic review of randomized controlled clinical trials of abdominal acupuncture treatment of cervical spondylosis [Chinese] 2011; 36: 137-44. [abstract only]

\section{Exclude for Thermal}

Intervention (Multimodal, Thermal Agent Part of Cluster Treatment - Dosage and/or Contribution of Each Unclear)

Brønfort G, Nilsson N, Haas M, Evans RL, Goldsmith $\mathrm{CH}$, Assendelft WJJ, Bouter LM. Non-invasive physical treatments for chronic/recurrent headache. Cochrane Database Syst Rev 2004; 3: CD001878.

Haraldsson BG, Gross AR, Myers CD, Ezzo JM, Morien A, Goldsmith C, et al. Massage for mechanical neck disorders. [Review] [114 refs]. Cochrane Database Syst Rev 2006; 3: CD004871.

Kay TM, Gross A, Goldsmith CH, Hoving JL, Bronfort G. Exercises for mechanical neck disorders. Cochrane Database Syst Rev 2009; (4).

Miller J, Gross A, D’Sylva J, Burnie SJ, et al. Manual therapy and exercise for neck pain: A systematic review Man Ther 2010; 15: 334-54.

Verhagen AP, Scholten-Peeters GGGM, van Wijngaarden S, de Bie R, Bierma-Zeinstra SMA. Conservative treatments for whiplash. Cochrane Database of Systematic Reviews 2007; 2: CD003338.

Vernon H, Humphreys K, Hagino C. Chronic mechanical neck pain in adults treated by manual therapy: a systematic review of change scores in randomized controlled trials. J Manipulative Physiol Ther 2007; 30: 215-27.

\section{Comparison (Thermal Agent was Active Control or Co- Intervention)}

Conlin A, Bhogal S, Sequeira A, Teasell R. Treatment of whiplash-associated disorders-Part 1: Non-invasive interventions. Pain Res Manage 2005; 10(1): 21-32.

Haines T, Gross A, Burnie SJ, Goldsmith CH, Perry L. Patient education for neck pain with or without radiculopathy. [Review] [123 refs][Update of Cochrane Database Syst Rev 2008; (4): CD005106; PMID: 18843681]. Cochrane Database Syst Rev 2009; (1): CD005106.

Trinh K, Graham N, Gross A, et al. Acupuncture for neck disorders. Spine 2007; 32(2): 236-43.

\section{Design}

Tsakitzidis G, Remmen R, Peremans L, et al. Nonspecific neck pain: diagnosis and treatment. Good Clinical Practice (GCP). Brussels: Belgian Health Care Knowledge Centre (KCE); 2009. Report No.: KCE Reports 119C.

Whiffen P. Evidence-based pain management and palliative care in Issue 3 for 2006 of the Cochrane Library. J Pain Palliat Care Pharmacother 2007; 21(1): 53-6.

\section{Low AMSTAR Rating}

Hurwitz EL, Carragee EJ, van der Velde G, et al. Treatment of neck pain; noninvasive interventions: results of 
the Bone and Joint Decade 2000-2010 Task Force on Neck Pain and Its Assoiciated Disorders. Spine 2008; 33(4 Suppl); S123-S152.

Peake N, Harte A. The effectiveness of cervical traction. Phys Ther Rev 2005; 10: 217-29.

Teasell RW, McClure JA, Walton D, Pretty J, Salter K, etaL A research synthesis of therapeutic interventions for whiplash-associated disorders (WAD): Part 3-Interventions for WAD. Pain Res Manage 2010; 15(5): 305-12.

Vernon HT, Humphreys BK, Hagino CA. A systematic review of conservative treatments for acute neck pain not due to whiplash. J Manipulative Physiol Ther 2005; 28(6): 443-8.

Vernon H, Humphreys BK, Hagino C. The outcome of control groups in clinical trials of conservative treatments for chronic mechanical neck pain: A systematic review. BMC Musculoskeletal Disorders 2006; 7: 58.

Vernon H, Schneider M. Chiropractic management of myofascial trigger points and myofascial pain syndrome: A systematic review of the literature [review] J Manipulative Physiol Ther 2009; 32(1): 14-24.

Exclude for Traction

\section{Intervention (Manual Traction)}

Gross A, Miller J, D'Sylva J, Burnie SJ, et al. Manipulation or mobilisation for neck pain. [Review] [173 refs][Update of Cochrane Database Syst Rev 2004; (1): CD004249; PMID: 14974063]. Cochrane Database Syst Rev 2010; (1): CD004249.

Kay TM, Gross A, Goldsmith CH, Hoving JL, Bronfort G. Exercises for mechanical neck disorders. Cochrane Database Syst Rev 2009; (4).

Kroeling P, Gross A, Goldsmith CH, Burnie SJ, Haines $\mathrm{T}$, Graham N, et al. Electrotherapy for neck pain. [Review] [73 refs][Update of Cochrane Database Syst Rev 2005; (2): CD004251; PMID: 15846703]. Cochrane Database Syst Rev 2009; (4): CD004251.

Kroeling $\mathrm{P}$, Gross A, Goldsmith $\mathrm{CH}$, et al. Electrotherapy for neck pain. Cochrane Database Syst Rev 2012.

Miller J, Gross A, D’Sylva J, Burnie SJ, et al. Manual therapy and exercise for neck pain: A systematic review. Man Ther 2010; 15: 334-54.

Williams NH, Hendry M, Lewis R, Russell I, Westmoreland A, Wilkinson C. Psychological response in spinal manipulation (PRISM): a systematic review of psychological outcomes in randomised controlled trials. [Review] [50 refs]. Complement Ther Med 2007; 15(4): 271-83.

\section{Low AMSTAR Rating}

Hurwitz EL, Carragee EJ, van der Velde G, et al. Treatment of neck pain; noninvasive interventions: results of the Bone and Joint Decade 2000-2010 Task Force on Neck Pain and Its Assoiciated Disorders. Spine 2008; 33(4 Suppl); S123-S152.
Peake N, Harte A. The effectiveness of cervical traction. Phys Ther Rev 2005; 10: 217-29.

Exclude for Electrotherapy

\section{Intervention}

Brønfort G, Nilsson N, Haas M, Evans RL, Goldsmith $\mathrm{CH}$, Assendelft WJJ, Bouter LM. Non-invasive physical treatments for chronic/recurrent headache. Cochrane Database Syst Rev 2004; 3: CD001878. DOI: 10.1002/14651858.CD001878.pub2.

Haines T, Gross A, Burnie SJ, Goldsmith CH, Perry L. Patient education for neck pain with or without radiculopathy. [Review] [123 refs][Update of Cochrane Database Syst Rev 2008; (4): CD005106; PMID: 18843681]. Cochrane Database Syst Rev 2009; (1): CD005106.

Haraldsson BG, Gross AR, Myers CD, Ezzo JM, Morien A, Goldsmith C, et al. Massage for mechanical neck disorders. [Review] [114 refs]. Cochrane Database Syst Rev 2006; 3: CD004871.

\section{Design}

Tsakitzidis G, Remmen R, Peremans L, Van Royen P, Duchesnes C, Paulus D, et al. Non-specific neck pain: diagnosis and treatment. Good Clinical Practice (GCP). Brussels: Belgian Health Care Knowledge Centre (KCE) 2009; Report No.: KCE Reports 119C.

\section{Low AMSTAR Rating}

Hurwitz EL, Carragee EJ, van der Velde G, et al. Treatment of neck pain; noninvasive interventions: results of the Bone and Joint Decade 2000-2010 Task Force on Neck Pain and Its Assoiciated Disorders. Spine 2008; 33(4 Suppl); S123-S152.

Lee MS, Choi T-Y, Kim J-I, Choi S-M. Using Guasha to treat musculoskeletal pain: A systematic review of controlled clinical trials. Chinese Medicine 2010; 5: 5.

Teasell RW, McClure JA, Walton D, et al. A research synthesis of therapeutic interventions for whiplashassociated disorders (WAD): Part 2-Interventions for WAD. Pain Res Manage 2010; 15(5): 295-304.

Vernon HT, Humphreys BK, Hagino CA. A systematic review of conservative treatments for acute neck pain not due to whiplash. J Manipulative Physiol Ther 2005; 28(6): 443-8.

Vernon H, Humphreys BK. Manual therapy for neck pain: an overview of randomized clinical trials and systematic reviews. [Review] [141 refs]. Europa Medicophysica 2007 Mar; 43(1): 91-118.

Vernon H, Schneider M. Chiropractic management of myofascial trigger points and myofascial pain syndrome: A systematic review of the literature [review]. J Manipulative Physiol Ther 2009; 32(1): 14-24.

Wang S-Z, Lin H-L, Song H-M, et al. Conservative in the treatment of protrusion of cervical vertebra intervertebral disc: A systematic review. [Chinese]. J Evid Based Med 2009; 9(3): 331-6. 


\section{REFERENCES}

[1] Makela M, Heliovaara M, Sievers K, Impivaara O, Knekt P, Aromaa A. Prevalence, determinants, and consequences of chronic neck pain in Finland. Am J Epidemiol 1991; 134(11): 1356-67.

[2] Rajala M. Health promotion policies, strategies and structures. Promot Educ 1995; 2(2-3): 12-3.

[3] Cote P, Cassidy JD, Carroll L. The Saskatchewan Health and Back Pain Survey. The prevalence of neck pain and related disability in Saskatchewan adults. Spine (Phila Pa 1976 ) 1998; 23(15): 168998.

[4] Chiu TT, Leung AS. Neck pain in Hong Kong: a telephone survey on prevalence, consequences, and risk groups. Spine 2006; 31(16): E540-4.

[5] Fejer R, Kyvik KO, Hartvigsen J. The prevalence of neck pain in the world population: a systematic critical review of the literature. Eur Spine J 2006; 15(6): 834-48.

[6] Guez M, Hildingsson C, Nasic S, Toolanen G. Chronic low back pain in individuals with chronic neck pain of traumatic and nontraumatic origin: A population-based study. Acta Orthop 2006; 77(1): 132-7.

[7] Hogg-Johnson S, van der Valde G, Carroll LJ, et al. The burden and determinants of neck pain in the general population: results of the Bone and Joint Decade 2000-2010 Task Force on Neck Pain and Its Associated Disorders. Spine (Phila Pa 1976 ) 2008; 33(4 Suppl): S39-S51.

[8] Riddle DL, Schappert SM. Volume and characteristics of inpatient and ambulatory medical care for neck pain in the United States: data from three national surveys. Spine (Phila Pa 1976 ) 2007; 32(1): 132-40.

[9] Borghouts J, Janssen H, Koes B, Muris J, Metsemakers J, Bouter L. The management of chronic neck pain in general practice. A retrospective study. Scand J Prim Health Care 1999; 17(4): 215-20.

[10] Hoving JL, Koes BW, De Vet HCW, et al. Manual therapy, physical therapy or continued care by the general practitioner for patients with neck pain: short-term results from a pragmatic randomized trial. In: Hoving JL, Ed. Neck pain in primary care: the effects of commonly applied interventions. Netherlands: Vrije Universiteit; 2001; pp. 43-57.

[11] Cote P, Vandervelde G, Cassidy JD, et al. The burden and determinants of neck pain in workers: results of the Bone and Joint Decade 2000-2010 Task Force on Neck Pain and Its Associated Disorders. Spine (Phila Pa 1976 ) 2008; 33(4 Suppl): S60-S74.

[12] Gross AR, Aker PD, Goldsmith CH, Peloso P. Conservative management of mechanical neck disorders. A systematic overview and meta-analysis. Online J Curr Clin Trials 1996; 200-201: 34457.

[13] Verhagen AP, Scholten-Peeters GGGM, Van Wijngaarden S, de Bie RA, Bierma-Zeinstra SMA. Conservative treatments for whiplash. Cochrane Database Syst Rev 2007; (2): CD003338.

[14] Gross AR, Kay T, Hondras M, et al. Manual therapy for mechanical neck disorders: a systematic review. Man Ther 2002; 7(3): 131-49.

[15] Spitzer W, Skovron M, Salmi L, et al. Scientific Monograph of the Quebec Task Force on Whiplash-Associated Disorders: Redefining "Whiplash" and Its Management. Spine 1995; 20(8S): 1S-73S.

[16] Gross AR, Goldsmith C, Hoving JL, et al. Conservative management of mechanical neck disorders: a systematic review. [Review] [137 refs]. J Rheumatol 2007; 34(5): 1083-102.

[17] Rickards LD. The effectiveness of non-invasive treatments for active myofascial trigger point pain: A systematic review of the literature. Int J Osteop Med 2006; 9(4): 120-36.

[18] Vernon HT, Humphreys BK, Hagino CA. A systematic review of conservative treatments for acute neck pain not due to whiplash. J Manipulative Physiol Ther 2005; 28(6): 443-8.

[19] Kroeling P, Gross A, Goldsmith $\mathrm{CH}$, et al. Electrotherapy for neck pain. [Review] [73 refs][Update of Cochrane Database Syst Rev. 2005; (2): CD004251; PMID: 15846703]. Cochrane Database Syst Rev 2009; (4): CD004251.

[20] Gross AR, Aker PD, Goldsmith CH, Peloso P. Physical medicine modalities for mechanical neck disorders [Systematic Review]. Cochrane Database of Systematic Reviews Physical medicine modalities for mechanical neck disorders [Systematic Review]. Cochrane Database Syst Rev 2000; (2): CD000961.

(21] Graham N, Gross AR, Goldsmith C, Cervical Overview Group. Mechanical traction for mechanical neck disorders: a systematic review. [Review] [37 refs]. J Rehabil Med 2006; 38(3): 145-52.
Kjellman GV, Skargren EI, Oberg BE. A critical analysis of randomised clinical trials on neck pain and treatment efficacy. A review of the literature. Scand J Rehabil Med 1999; 31(3): 139-52.

[23] Peeters GGM, Verhagen AP, de Bie RA, Oostendorp RAB. The efficacy of conservative treatment in patients with whiplash injury: a systematic review of clinical trials. Spine 1915; 26(4): E64-E73.

[24] van der Heijden GJ, Beurskens AJ, Koes BW, Assendelft WJ, de Vet HC, Bouter LM. The efficacy of traction for back and neck pain: a systematic, blinded review of randomized clinical trial methods. Phys Ther 1995; 75(2): 93-104.

[25] Fu L, Li J, Wu W. Randomized controlled trials of acupuncture for neck pain: systematic review and meta-analysis. J Alternat Complement Med 2009; 15(2): 133-45.

[26] Furlan AD, Yazdi F, Tsertsvadze A, et al. A systematic review and meta-analysis of efficacy, cost-effectiveness, and safety of selected complementary and alternative medicine for neck and low-back pain. Evid Based Complement Alternat Med 2012; 2012: 953139.

[27] Trinh K, Graham N, Gross A, et al. Acupuncture for neck disorders. Spine 2007; 32(2): 236-43.

[28] Vernon H, Schneider M. Chiropractic management of myofascial trigger points and myofascial pain syndrome: A systematic review of the literature [review]. J Manipulative Physiol Ther 2009; 32(1): 14-24.

[29] Michlovitz S. Modalities for Therapeutic Intervention. Philadelphia: F.A. Davis Company 2005.

[30] Tan JC, Nordin M. Role of physical therapy in the treatment of cervical disk disease. Orthop Clin North Am 1992; 23(3): 435-49.

[31] Lehmann JF. Therapeutic Heat and Cold. $4^{\text {th }}$ ed. Baltimore: Williams \& Wilkins 1990.

[32] Tollison CD, Satterthwaite JR. Painful cervical trauma: diagnosis and rehabilitative treatment of neuromusculoskeletal injuries. Philadelphia: Williams \& Wilkins 1992.

[33] Crumley M, Nowak P, Merrick M. Do ultrasound, active warm-up and passive motion differ on their ability to cause temperature and range of motion changes? J Athl Train 2001; 36(2S): S-92.

[34] Prentice W, Quillen W. Therapeutic Modalities in Rehabilitation. New York: McGraw-Hill 2005.

[35] Bashardoust TS, Houghton P, MacDermid JC, Grewal R. Effects of low-intensity pulsed ultrasound therapy on fracture healing: a systematic review and meta-analysis. Am J Phys Med Rehabil 2012; 91(4): 349-67.

[36] Kisner C, Colby LA. The spine: traction procedures. Therapeutic exercise: foundations and techniques. $3^{\text {rd }}$ ed. Philadelphia: F.A. Davis Co 1996; pp. 575-91.

[37] Michelle HC. Physical agents in rehabilitation. From research to practice. $1^{\text {st }}$ ed. Philadelphia, New York, London: WB Saunders Company 1999.

[38] Hsueh TC, Cheng PT, Kuan TS, Hong CZ. The immediate effectiveness of electrical nerve stimulation and electrical muscle stimulation on myofascial trigger points. Am J Phys Med Rehabil 1997; 76(6): 471-6.

[39] Stux G, Pomeranz B. Stux G, Pomeranz B. Basics of Acupuncture, 3rd ed. Berlin: Springer-Verlag, 1995. $3^{\text {rd }}$ ed. Berlin: SpringerVerlag 2012.

[40] Pomeranz B. Acupuncture analgesia-basic research. In: Stux G, Hammerschlag R, Eds. Clinical acupuncture: scientific basis. Germany: Springer 2001; pp. 1-28.

[41] Liangyue D, Yijun G, Shuhui H, et al. Chinese Acupuncture and Moxibustion. 1987 ed. Beijing: Foreign Languages Press 1987.

[42] Trinh K. The efficacy of acupuncture in acute non-specific low back pain and sciatica: A randomized controlled trial in primary care. Thesis. Hamilton, Ontario: McMaster University 2000.

[43] Davis C. Complementary Therapies in Rehabilitation: Evidence for Efficacy in Therapy, Prevention and Wellness. $3^{\text {rd }}$ ed. New Jersey: SLACK Incorporated 2009.

[44] Kenneth K, Draper D. Therapeutic Modalities: the art and the science. Philadelphia: Lippincott Williams \& Wilkins 2008.

[45] Bashardoust TS, MacDermid JC, Houghton P, Grewal R. Effects of low power laser irradiation on bone healing in animals: a metaanalysis. J Orthop Surg Res 2010; 5: 1.

[46] Maeda T. Morphological demonstration of low reactive laser therapeutic pain attenuation effect of the Gallium Aluminium Arsenide diode laser. Pain 1988; 72: 25-30.

[47] Belanger A. Laser. Evidence-based guide to therapeutic physical agents. Philadelphia: Lippincott Williams \& Wilkins 2003; pp. 191-221. 
[48] Trelles MA, Mayayo E, Miro L, Rigau J, Baudin G, Calerhead RG. The action of low reactive level laser therapy (LLLT) on mast cells: a possible pain relief mechanism examined. J Orthop Sports Phys Ther 2003; 4: 27-30.

[49] Goldsmith CH, Gross AR, MacDermid J, Santaguida L, Miller J. What does the evidence tell us about design of future treatment trials for whiplash-associated disorders? Spine 2011; 36(25S): S292-S302.

[50] Verhagen AP, Peeters GGM, de Bie RA, Oostendorp RAB. Conservative treatment for whiplash (Cochrane Review). Cochrane Database Syst Rev 2004; (1): CD003338.

[51] Hurwitz EL, Carragee EJ, van d, V, et al. Treatment of neck pain: noninvasive interventions: results of the Bone and Joint Decade 2000-2010 Task Force on Neck Pain and Its Associated Disorders. Spine (Phila Pa 1976 ) 2008; 33(4 Suppl): S123-S152.

[52] Holm LW, Carroll LJ, Cassidy JD, et al. The burden and determinants of neck pain in whiplash-associated disorders after traffic collisions: results of the Bone and Joint Decade 2000-2010 Task Force on Neck Pain and Its Associated Disorders. Spine (Phila Pa 1976 ) 2008; 33(4 Suppl): S52-S59.

[53] Linton SJ, Hellsing AL, Hallden K. A population-based study of spinal pain among 35-45-year-old individuals. Prevalence, sick leave, and health care use. Spine (Phila Pa 1976 ) 1998; 23(13): 1457-63.

[54] Shea BJ, Hamel C, Wells GA, et al. AMSTAR is a reliable and valid measurment tool to assess the methodological quality of systematic reviews. J Clin 2009; 62(10): 1013-20.

[55] Balshem $\mathrm{H}$, Helfand $\mathrm{M}$, Schunemann HJ, et al. GRADE guidelines: 3. Rating the quality of evidence. J Clin Epidemiol 2011; 64(4): 401-6.

[56] Guyatt GH, Oxman A, Vist G. GRADE guidelines: 4. Rating the quality of evidence--study limitations (risk of bias). J Clin Epidemiol 2011; 64(4): 407-15.

[57] Santaguida L, MacDermid J, Gross A, et al. International Collaboration on Neck (ICON) methods report. 2012.

[58] Whitlock EP, Lin JS, Chou R, Shekelle P, Robinson KA. Using Existing Systematic Reviews in Complex Systematic Reviews. Ann Intern Med 2008; 148: 776-82.

[59] Jadad AR, Moore RA, Carroll D, et al. Assessing the quality of reports of randomized clinical trials: is blinding necessary? Control Clin Trials 1996; 17: 1-12.

[60] Sherrington C, Herbert R D, Maher CG, Moseley AM. PEDro. A database of randomized trials and systematic reviews in physiotherapy. Man Ther 2000; 5(4): 223-6.

[61] Birch SJ. Controlled trial of Japanese acupuncture for chronic myofascial neck pain: assessment of specific and nonspecific effects of treatment. Clin J Pain 1998; 14(3): 248-55.

[62] Nabeta T, Kawakita K. Relief of chronic neck and shoulder pain by manual acupuncture to tender points--a sham-controlled randomized trial. Complement Ther Med 2002; 10(4): 217-22.

[63] Zhu XM, Polus B. A controlled trial on acupuncture for chronic neck pain. Am J Chin Med 2002; 30(1): 13-28.

[64] White PF, Craig WF, Vakharia AS, Ghoname E, Ahmed HE, Hamza MA. Percutaneous neuromodulation therapy: does the location of electrical stimulation effect the acute analgesic response? Anesth Analg 2000; 91(4): 949-54.

[65] Irnich D, Behrens $\mathrm{N}$, Molzen $\mathrm{H}$, et al. Randomised trial of acupuncture compared with conventional massage and "sham" laser acupuncture for treatment of chronic neck pain. BMJ 2001; 322: 1574-7.

[66] Irnich D, Behrens N, Gleditsch JM, et al. Immediate effects of dry needling and acupuncture at distant points in chronic neck pain: results of a randomized, double-blind, sham-controlled crossover trial. Pain 2002; 99(1-2): 83-9.

[67] Petrie JP, Langley GB. Acupuncture in the treatment of chronic cervical pain. A pilot study. Clin Exp Rheumatol 1983; 1(4): 333-6.

[68] White P, Lewith G, Prescott P, Conway J. Acupuncture versus placebo for the treatment of chronic mechanical neck pain: a randomized, controlled trial. Ann Intern Med 2004; 141(12): 911-9.

[69] Coan RM, Wong G, Coan PL. The acupuncture treatment of neck pain: a randomized controlled study. American Journal of Chinese Medicine. Am J Chin Med 1981; 9: 326-32.

[70] Goldie I, Landquist A. Evaluation of the effects of different forms of physiotherapy in cervical pain. Scand J Rehabil Med 1970; 2(2): 117-21.
[71] Zylbergold RS. Cervical spine disorders. A comparison of three types of traction. Spine 1985; 10(10): 867-71.

[72] Ceccherelli F, Altafini L, Lo CG, Avila A, Ambrosio F, Giron GP Diode laser in cervical myofascial pain: a double-blind study versus placebo. Clin J Pain 1989; 5(4): 301-4.

[73] Chow RT, Heller GZ, Barnsley L. The effect of $300 \mathrm{~mW}, 830 \mathrm{~nm}$ laser on chronic neck pain: a double-blind, randomized, placebocontrolled study. Pain 2006; 124(1-2): 201-10.

[74] Ozdemir F, Birtane M, Kokino S. The clinical efficacy of lowpower laser therapy on pain and function in cervical osteoarthritis. Clin Rheumatol 2001; 20(3): 181-4.

[75] Soriano F, Rios R, Pedrola M, Giagnorio J, Battagliotti C. Acute cervical pain is relieved with gallium-arsenida (GaAs) laser irradiation. A double-blind preliminary study. Laser Ther 1996; 8: 149-54.

[76] Taverna E, Parrini M, Cabitza P. Laser therapy $v s$ placebo in the treatment of some bone and joints pathology. Minerva Ortop 1997; 41: 631-6.

[77] Edwards J, Knowles N. Superficial dry needling and active stretching in the treatment of myofascial pain--a randomised controlled trial. Acupunct Med 2003; 21(3): 80-6.

[78] Loy TT. Treatment of cervical spondylosis. Electroacupuncture versus physiotherapy. Med J Aust 1983; 2: 32-4.

[79] Guangyue W, Fenglin Q. Treatment of 482 cases of cervical spondylopathy by combining point-injection and needle-warming via moxibustion. J Tradit Chin Med 2001; 21: 31-3.

[80] Zhou JW, Jiang ZY, Ye RB, et al. Controlled study on treatment of cervical spondylopathy of the nerve root type with acupuncture, moxibustion and massage as main. Zhongguo Zhen Jiu 2006; 26(8): 537-43.

[81] Fu WB, Zhang HL, Fan L. Treatment of cervical spondylopathy by needle pricking: a clinical observation of 56 cases. J Tradit Chin Med 2005; 37: 65-6.

[82] Wang C, Xiong Z, Deng C, Yu W, Ma W. Miniscalpel-needle versus trigger-point injection for cervical myofascial pain syndrome: A randomized comparative trial J Alternat Complement Med 2007; 13(1): 14-6.

[83] Esenyel M. Treatment of myofascial pain. Am J Phys Med Rehabil 2000; 79(1): 48-52.

[84] Majlesi J, Unalan H. High-power pain threshold ultrasound technique in the treatment of active myofascial trigger points: a randomized, double-blind, case-control study. Arch Phys Med Rehabil 2004; 85(5): 833-6.

[85] Ilbuldu E, Cakmak A, Disci R, Aydin R. Comparison of laser, dry needling, and placebo laser treatments in myofascial pain syndrome. Photomed Laser Surg 2004; 22(4): 306-11.

[86] Konstantinovic L, Cutovic M, Milovanovic A, et al. Low-level laser therapy for acute neck pain with radiculopathy: A doubleblind placebo-controlled randomized study. Pain Med 2010; 11: 1169-78.

[87] Gur A, Sarac, Cevik R, Altindag O, Sarac S. Efficacy of 904nm gallium arsenide low level laser therapy in the management of chronic myofascial pain in the neck: a couble-blind and randomized-controlled trial. Lasers Surg Med 2004; 35: 229-35.

[88] Smania N, Corato E, Fiaschi A, Pietropoli P, Aglioti SM, Tinazzi M. Repetitive magnetic stimulation: a novel therapeutic approach for myofascial pain syndrome. J Neurol 2005; 252(3): 307-14.

[89] Flynn T. A comparative study between ultrareiz and ultrasound in the treatment for relief of pain in whiplash injuries. Physiother Irel 1987; 8(1): 11-4.

[90] Henriks O, Horgan A. Ultra-reiz current as an adjunct to standard physiotherapy treatment of the acute whiplash patient. Physiother Ireland 1996; 17(1): 13-7.

[91] Gam AN, Warming S, Larsen LH, et al. Treatment of myofascial trigger-points with ultrasound combined with massage and exercise-a randomised control led trial. Pain 1998; 77: 73-9.

[92] Coppieters MW, Stappaerts KH, Wouters LL, Janssens K. The immediate effects of a cervical lateral glide treatment technique in patients with neurogenic cervicobrachial pain. J Orthop Sports Phys Ther 2003; 33(7): 369-78.

[93] Hurwitz EL, Morgenstern H, Harber P, Kominski GF, Yu F, Adams AH. A randomized trial of chiropractic manipulation and mobilization for patients with neck pain: clinical outcomes from the UCLA neck-pain study. Am J Public Health 2002; 92(10): 1634-41. 
[94] Lewith GT, Machin D. A randomized trial to evaluate the effect of infra-red stimulation of local trigger points, versus placebo, on the pain caused by cervical osteoarthrosis. Res Int J 1981; 6: 277-84.

[95] Pain in the neck and arm: a multicentre trial of the effects of physiotherapy, arranged by the British Association of Physical Medicine. BMJ 1966; 5482: 253-8.

[96] Klaber M, Jackson DA, Gardiner ED, et al. Randomized trial of two physiotherapy interventions for primary care neck and back pain patients: 'McKenzie' vs brief physiotherapy pain management. Rheumatology 2006; 45(12): 1514-21.

[97] Giles LG, Muller R. Chronic spinal pain syndromes: a clinical pilot trial comparing acupuncture, a nonsteroidal anti-inflammatory drug, and spinal manipulation. J Manipulative Physiol Ther 1999; 22: $376-81$

[98] Thomas M, Eriksson SV, Lundeberg T. A comparative study of diazepam and acupuncture in patients with osteoarthritis pain: a placebo controlled study. Am J Chin Med 1991; XIX(2): 95-100.

[99] Muller R, Giles LG. Long-term follow-up of a randomized clinical trial assessing the efficacy of medication, acupuncture, and spinal manipulation for chronic mechanical spinal pain syndromes. J Manipulative Physiol Ther 2005; 28(1): 3-11.

[100] Lee JC, Lin DT, Hong C. The effectiveness of simultaneous thermotherapy with ultrasound and electrotherapy with combined AC and DC current on the immediate pain relief of myofascial trigger points. J Musculoskelet Pain 1997; 5(1): 81-90.

[101] Hou C, Tsai L, Cheng K, Chung K, Hong C. Immediate effects of various physical therapeutic modalities on cervical myofascial pain and trigger-point sensitivity. Arch Phys Med Rehabil 2002; 83(10): 1406-14.

[102] Snow CJ, Aves Wood R, Dowhopoluk V, Howdle HDC, Elfenbaum G. Randomized controlled clinical trial of spray and stretch for relief for back and neck myofascial pain. Physiother Can 1992; 44: S8.

[103] Waylonis GW, Wilke S, O'Toole D, Waylonis DA, Waylonis DB. Chronic myofascial pain: management by low-output helium-neon laser therapy. Arch Phys Med Rehabil 1988; 69(12): 1017-20.

[104] Nilsson N. A randomized controlled trial of the effect of spinal manipulation in the treatment of cervicogenic headache. J Manipulative Physiol Ther 1995; 18(7): 435-40.

[105] Seidel U, Ulhemann C. Behandelung der zervikalen tendomyosis. Randomisierte, kontrollierte doppelblindstudie zur dosierten lasertherapie an akupunkturpunkten und classischen akupunktur. Dtsch Z Akupunkt 2002; 2: 258-69.

[106] Philipson T. [The effect of diadynamic current on chronic softtissue pain in the neck and shoulder girdle]. [Danish]. Ugeskr Laeger 1983; 145(7): 479-81.

[107] Fialka V, Preisinger E, Bohler A. Zur physikalischen Diagnostik und physikalischen Therapie der Distorsio columnae vertebralis cervicalis. Z Phys Med Baln Med Klim 1989; 18: 390-7.

[108] Chiu TW, Hui-Chan C, Cheing G. A randomized clinical trial of TENS and exercise for patients with chronic neck pain. Clin Rehabil 2005; 19: 850-60.

[109] Nordemar R, Thorner C. Treatment of acute cervical pain- a comparative group study. Pain 1981; 10: 93-101.

[110] Escortell ME. Primary care randomized clinical trial: Manual therapy effectivenss in comparison with TENS in patients with neck pain. Man Ther 2011; 16: 66-73.

[111] Farina S, Casarotto M, Benelle M, et al. A randomized controlled study on the effect of two different treatments in myofascial pain syndrome. Eur Med Phys 2004; 40: 293-301.

[112] Thorsen H. [Low energy laser treatment--effect in localized fibromyalgia in the neck and shoulder regions]. [Danish]. Ugeskr Laeger 1991; 153(25): 1801-4.

[113] Ceccherelli F, Altafini L, Lo Castro G, Avila A, Ambrosio F, Giron GP. Diode laser in cervical myofascial pain: A double-blind study versus placebo. Clin J Pain 1989; 5: 301-4.

[114] Thorsen H. Low level laser therapy for myofascial pain in the neck and shoulder girdle. A double-blind, cross-over study. Scand J Rheumatol 1992; 21(3)139-41.

[115] Goldsmith CH, Gross AR, MacDermid J, Santaguida L, Miller J. What does the evidence tell us about design of future treatment trials for whiplash-associated disorders? Spine 2011; 36(25S): S292-S302.

[116] Moher D. CONSORT: an evolving tool to help improve the quality of reports of randomized controlled trials. Consolidated Standards of Reporting Trials. JAMA 1998; 279: 1489-91.
[117] Schultz K, Altman D, Moher D. CONSORT statement: updated guidelines for reporting parallel group randomised trials. BMJ 2010; 340: 698 .

[118] Jette A. Toward a Common Language for Function, Disability, and Health. Phys Ther 2006; 86: 726-34.

[119] Carlesso LC, Cairney J, Dolovich L, Hoogenes J. Defining adverse events in manual therapy: An exploratory qualitative analysis of the patient perspective. Man Ther 2011; 16(5): 440-6.

[120] Carlesso LC, MacDermid JC, Santaguida L. Standardization of adverse event terminology and reporting in orthopaedic physical therapy-applications to the cervical spine. Spine 2010; 40: 455-63.

[121] Rajendran D, Mullinger B, Fossum C. Monitoring self-reported adverse events: a prospective, pilot study in a UK osteopathic teaching clinic. Int J Osteop Med 2009; 12: 49-55.

[122] Wheway J, Agbabiaka TB, Ernst E. Patient safety incidents from acupuncture treatments: a review of reports to the National Patient Safety Agency. Int J Risk Saf Med 2012; 24(3): 163-9.

[123] Ernst E, Lee MS, Choi TY. Acupuncture: does it alleviate pain and are there serious risks? A review of reviews. Pain 2011; 152(4): 755-64.

[124] Hahne AJ, Ford JJ, McMeeken JM. Conservative management of lumbar disc herniation with associated radiculopathy: A systematic review. Spine 2010; 35(11): E488-504.

[125] Sackett D, Rosenberg M, Muir Gray J, Haynes B, Richardson W. Evidence based medicine: what it is and what it isn't. BMJ 1996; 312: 71-2.

[126] Kroeling P, Gross A, Goldsmith CH, et al. Electrotherapy for neck pain. Cochrane Database Syst Rev 2013 [Epub ahead of print].

[127] Thuile $\mathrm{CH}$, Walzi M. Evaluation of electromagnetic fields in the treatment of pain in patients with lumbar radiculopathy or the whiplash syndrome. Neurorehabilitation 2002; 17: 63-7.

[128] Sutbeyaz ST, Sezer N, Koseoglu BF. The effect of pulsed electromagnetic fields in the treatment of cervical osteoarthritis: a randomized, double-blind, sham-controlled trial. Rheumatol Int 2006; 26(4): 320-4.

[129] Trock DH. The effect of pulsed electromagnetic fields in the treatment of osteoarthritis of the knee and cervical spine. Report of randomized, double blind, placebo controlled trials. J Rheumatol 1994; 21(10): 1903-11.

[130] Foley-Nolan D, Moore K, Codd M, Barry C, O'Connor P, Coughlan RJ. Low energy high frequency pulsed electromagnetic therapy for acute whiplash injuries. A double blind randomized controlled study. Scand J Rehabil Med 1992; 24(1): 51-9.

[131] Foley KT, Mroz TE, Arnold PM, et al. Randomized, prospective, and controlled clinical trial of pulsed electromagnetic field stimulation for cervical fusion. Spine J 2008; 8(3): 436-42.

[132] Conlin A, Bhogal S, Sequeira K, Teasell R. Treatment of whiplashassociated disorders--part I: Non-invasive interventions. Pain Res Manag 2005; 10(1): 21-32

[133] Smania N, Corato E, Fiaschi A, Pietropoli P, Aglioti SM, Tinazzi M. Therapeutic effects of peripheral repetitive magnetic stimulation on myofascial pain syndrome. Clin Neurophysiol 2003; 114(2): 350-8.

[134] Gross A. Low Level Laser Therapy (LLLT) for Neck Pain: A Systematic Review and Meta-Regression. In peer review. Clin Manag 2012 [Epub ahead of print].

[135] Chow RT, Barnsley L. Systematic Review of the Literature of Low-Level Laser Therapy (LLLT) in the Management of Neck Pain. Lasers Surg Med 2005; 37: 46-52.

[136] Chow RT, Johnson MI, Lopes-Martins RA, Bjordal JM. Efficacy of low-level laser therapy in the management of neck pain: systematic review and meta-analysis of randomised placebo or active-treatment controlled trials. [Review] [96 refs][Erratum appears in Lancet. 2010; 375(9718): 894]. Lancet 2009; 374(9705): 1897-908.

[137] Leaver AM, Refshauge K, Maher C, McAuley JH. Conservative interventions provide short-term relief for non-specific neck pain: a systematic review. J Physiother 2010; 56(2): 73-85.

[138] Graham N, Gross A, Goldsmith CH, et al. Mechanical traction for neck pain with or without radiculopathy. [Review] [59 refs]. Cochrane Database Syst Rev 2008; (3): CD006408.

[139] Kay TM, Gross A, Goldsmith CH, Hoving JL, Bronfort G Exercises for mechanical neck disorders. Cochrane Database Syst Rev 2012; 8: CD 004250.

[140] Graham N, Gross A, Goldsmith C, et al. Ultrasound for neck pain a systematic review. Physiother Can 2008; 61: 72-3. 
[141] Peloso PM, Gross A, Haines T, Trinh K, Goldsmith CH, Burnie S. Medicinal and injection therapies for mechanical neck disorders. Cochrane Database Syst Rev 2007; (3): CD 000319

[142] Nabeta T, Kawakita K. Relief of chronic neck and shoulder pain by manual acupuncture to tender points--a sham-controlled randomized trial. Complement Ther Med 2002; 10(4): 217-22.

[143] Fu L-M, Li J-T, Wu W-S. Randomized controlled trials of acupuncture for neck pain: Systematic review and meta-analysis. J Alternat Complement Med 2009; 15(2): 133-45.

[144] Trinh K, Graham N, Gross AR, et al. Acupuncture for Neck Disorders. Cochrane Database Syst Rev 2006; (4): CD 004870.

[145] White P, Lewith G, Prescott P, et al. Acupuncture versus placebo for the treatment of chronic mechanical neck pain. Ann Intern Med 2004; 141: 920-8.

[146] Haraldsson BG, Gross AR, Myers CD, et al. Massage for mechanical neck disorders. [Review] [114 refs]. Cochrane Database Syst Rev 2006; 3: CD004871.

[147] Furlan AD, Yazdi F, Tsertsvadze A, et al. A systematic review and meta-analysis of efficacy, cost-effectiveness, and safety of selected complementary and alternative medicine for neck and low-back pain. Evid Based Complement Alternat Med 2012; 2012: 953139.

[148] Zhou X, Bai Y-H. Combined therapy of traditional Chinese medicine and western medicine for low back pain. [Chinese]. Chin J Clin Rehabil 2006; 10(47): 118-20.

[149] Vernon H, Humphreys K, Hagino C. Chronic mechanical neck pain in adults treated by manual therapy: a systematic review of change scores in randomized clinical trials. J Manipulative Physiol Ther 2007; 30(3): 215-27.

[150] Gross A, Miller J, D'Sylva J, et al. Manipulation or mobilisation for neck pain. [Review] [173 refs][Update of Cochrane Database Syst Rev 2004; (1): CD004249; PMID: 14974063]. Cochrane Database Syst Rev 2010; (1): CD004249.
[151] Graham N, Gross A, Goldsmith C, Michlovitz S. Heat and cold for neck pain: a systematic review. Physiother Can 2009; 61: 73.

[152] Gross AR, Goldsmith C, Hoving JL, et al. Conservative management of mechanical neck disorders: a systematic review. J Rheumatol 2007; 34(5): 1083-102.

[153] Hong CZ, Lin JC, Bender LF, Schaeffer JN, Miltzer RJ, Causin P. Magnetic necklace: It's therapeutic effectiveness on neck and shoulder pain. Arch Phys Med Rehabil 1982; 63: 462-6.

[154] Chen L, Zhang X-L, Ding H, Tao Y-Q, Zhan H-S. Comparative study on effects of manipulation treatment and transcutaneous electrical nerve stimulation on patients with cervicogenic headache. [Chinese]. J Chin Integrat Med 2007; 5(4): 403-6.

[155] Waylonis G, Wilke S, O'Toole D, Waylonis D. Chronic myofascial pain: management by low-output helium-neon laser therapy. Arch Phys Med Rehabil 1988; 69: 1017-20.

[156] Aigner N, Fialka C, Radda C, Vecsei V. Adjuvant laser acupuncture in the treatment of whiplash injuries: A prospective, randomized placebo-controlled trial. [German]. Wien Klin Wochenschr 2006; 118(3-4): 95-9.

[157] David J, Modi S, Aluko AA, Robertshaw C. Chronic neck pain: a comparison of acupuncture treatment and physiotherapy. Br J Rheumatol 1998; 37: 1118-22.

[158] Altan L, Bingol U, Aykac M, Yurtkuran M. Investigation of the effect of GaAs laser therapy on cervical myofascial pain syndrome. Rheumatol Int 2005; 25(1): 25-7.

[159] Hakguder A, Birtane M, Gurcan S, Kokino S, Turan F. Efficacy of low level laser therapy in myofascial pain syndrome: An algometric and thermographic evaluation. Lasers Surg Med 2003; 33: 339-43.

[160] Dundar U, Evcik D, Samli F, Pusak H, Kavuncu V. The effect of gallium arsenide aluminum laser therapy in the management of cervical myofascial pain syndrome: a double blind, placebocontrolled study. Clin Rheumatol 2007; 26(6): 930-4. 